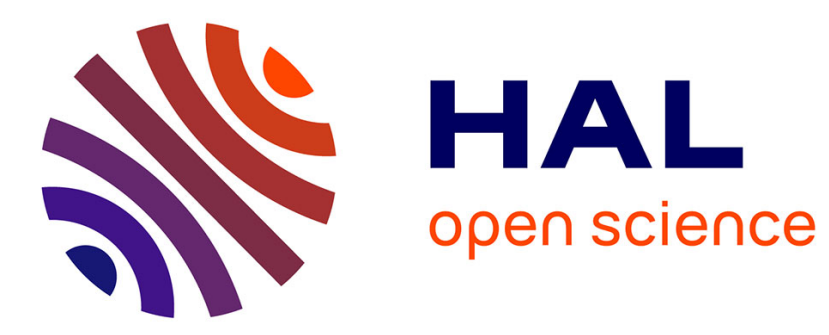

\title{
Effect of the measurement errors on two one-sided Shewhart control charts for monitoring the ratio of two normal variables
}

Huu Du Nguyen, Kim Phuc Tran

\section{- To cite this version:}

Huu Du Nguyen, Kim Phuc Tran. Effect of the measurement errors on two one-sided Shewhart control charts for monitoring the ratio of two normal variables. Quality and Reliability Engineering International, 2020, 10.1002/qre.2656 . hal-02562701

\section{HAL Id: hal-02562701 \\ https://hal.science/hal-02562701}

Submitted on 4 May 2020

HAL is a multi-disciplinary open access archive for the deposit and dissemination of scientific research documents, whether they are published or not. The documents may come from teaching and research institutions in France or abroad, or from public or private research centers.
L'archive ouverte pluridisciplinaire HAL, est destinée au dépôt et à la diffusion de documents scientifiques de niveau recherche, publiés ou non, émanant des établissements d'enseignement et de recherche français ou étrangers, des laboratoires publics ou privés. 


\title{
Effect of the measurement errors on two one-sided Shewhart control charts for monitoring the ratio of two normal variables
}

\author{
Huu Du Nguyen ${ }^{1}$ and Kim Phuc Tran*2 \\ ${ }^{1}$ Institute of Artificial Intelligence and Data Science, Dong A \\ University, Danang, Vietnam \\ ${ }^{2}$ Ecole Nationale Supérieure des Arts et Industries Textiles, \\ GEMTEX Laboratory, BP 3032959056 Roubaix Cedex 1, France, \\ *Corresponding author. Email: kim-phuc.tran@ensait.fr
}

April 1, 2020

\begin{abstract}
Monitoring the ratio between two random normal variables plays an important role in many industrial manufacturing processes. In this paper, we suggest designing two one-sided Shewhart control charts monitoring this ratio. The numerical results show that the one-sided charts have more advantages compared to the two-sided Shewhart chart proposed previously in the literature. Moreover, we investigate the effect of measurement error on the performance of these control charts where the measurement error is supposed to follow a linear covariate error model. The change of model parameters from an in-control condition to an out-of-control is presented without using a strict assumption about the independence of the shift size from measurement errors. A valuable finding from this study is that taking multiple measurements per item is not an effective way to reduce the negative effect of measurement error on the Shewhart charts' performance.
\end{abstract}

Keyword Shewhart control chart, Ratio distribution, Measurement error, One-sided chart, Linear covariate error.

\section{Introduction}

Designing a new control chart with higher performance in detecting shifts is a vital aim in many studies in Statistical Process Control (SPC), see, for example, Ren et al. ${ }^{1}$, Mukherjee and Rakitzis ${ }^{2}$, Ho et al. ${ }^{3}$, and Nguyen et al. ${ }^{4}$. However, a requirement of being easy and convenient to implement also 
plays an equally important role in deciding what kind of control chart to use. As a result, the Shewhart control chart introduced by Shewhart ${ }^{5}$ since 1931 is widely used in many industrial processes. Although the performance of the Shewhart chart in detecting small and moderate shifts is not as good as the one of the other advanced charts like the CUSUM control chart (Brook and Evans ${ }^{6}$ ) and the EWMA control chart (Hunter ${ }^{7}$ ), this kind of control chart has a major advantage of being easy to implement. Moreover, the Shewhart chart is well-known to be effective in detecting large process shifts. Consequently, it is usually considered first to monitor a process.

The ratio of two normal variables is a major concern in several industrial manufacturing processes. Several applications of this characteristic in practice have been pointed out in the literature. Tran et al. ${ }^{8}$ provided a real example in some battery recycling plants in Italy where the requirement of monitoring the ratio of "recyclable batteries" weight and "total batch" weight to quantify the economic loss is needed. Celano and Castagliola ${ }^{9}$ discussed another example in the food industry where the weight ratio between "pumpkin seeds" and "flaxseeds" is the characteristic of interest. Typical examples of the manufacturing environments that concern the ratio of two variables wewe mentioned in Tran et al. ${ }^{10},{ }^{11}$.

In order to enhance the practical use of the ratio chart, the effect of measurement error has been considered in recent times. In fact, the problem of measurement error has been investigated broadly in the literature, for example, Linna et al. ${ }^{12}$, Tang et al. ${ }^{13}$, Tran et al. ${ }^{14},{ }^{15},{ }^{16},{ }^{17}$, Cheng and Wang ${ }^{18}$, Sabahno et al. ${ }^{19}$, Nguyen et al. ${ }^{20}$, and Zaidi et al. ${ }^{21}$. For the case of the ratio control charts, Tran et al. ${ }^{8}$ was the first to study the effect of measurement error on a ratio Shewhart chart. However, in this study, the authors used a relatively tight assumption about the observed value of process shifts. That is, the observed process shift is independent from measurement error. In this study, we would like to reduce this assumption to make the study more practical. We use the same kind of control chart, which is the Shewhart chart, to monitor the ratio under the presence of measurement error. The effect of measurement error on the Shewhart ratio control chart will be explored without using this assumption. The change of parameters of the process in the presence of the measurement error will be discussed in detail.

Another problem that should be paid attention to in designing a control chart monitoring the ratio is the asymmetry of the ratio distribution. The distribution of a characteristic of interest is a deciding factor that influences the design of a control chart. For an asymmetric distribution, using a two-sided control chart to monitor both an increase and a decrease of the characteristic may lead to an $A R L$-biased (average run length) property in the sense that the out-of-control $A R L$ values are sometimes larger than the in-control value. This issue has been mentioned in several studies such as Nguyen et al. ${ }^{22},{ }^{23},{ }^{24}$, and Nguyen et al. ${ }^{25}$. The two-sided Shewhart control 
chart monitoring the ratio (say, the Shewhart-RZ control chart) suggested by Celano and Castagliola ${ }^{9}$ is also not an exception. An example of the $A R L$ biased situation witnessed in Celano and Castagliola ${ }^{9}$ will be illustrated in section 4 of this paper. Therefore, in this study, we suggest designing two one-sided Shewhart control charts to monitor separately an increase and a decrease in the ratio. The goal of this paper is then to investigate the performance of these one-sided control charts with or without the presence of measurement error. Under an assumption of no measurement error, the simulated results show that the one-sided Shewhart-RZ control charts perform better the two-sided Shewhart-RZ chart both in avoiding the $A R L$-biased problem and in detecting ratio shifts. Under the presence of measurement error, the effect of each parameter in the linear covariate error model on the charts' performance will be studied.

The rest of the paper is organized as follows. In section 2, we present briefly a review of the sample distribution of the ratio between two normal variables. Section 3 is to present the design and the implementation of the two-sided Shewhart-RZ control charts. The performance of these control charts in the case of no measurement error is given in section 4 . Section 5 demonstrates a linear covariate error model for the ratio and an establishment of the change in the model parameters from an in-control condition to an out-of-control one. The implementation and the design of the twosided Shewhart-RZ control charts in the presence of measurement error are discussed in section 6 while the effect of measurement error on the performance of these charts is presented in section 7. An illustrative example of the implementation of a two-sided Shewhart-RZ control chart under the appearance of measurement error is given in section 8 . Section 9 is for some concluding remarks.

\section{A brief review of the sample distribution the ratio}

Consider a bivariate normal random vector $\mathbf{W}=(X, Y)^{T}$ with the mean vector $\boldsymbol{\mu}_{\boldsymbol{W}}$ and the variance-covariance matrix $\boldsymbol{\Sigma}_{\boldsymbol{W}}$ defined by

$$
\boldsymbol{\mu}_{\boldsymbol{W}}=\left(\begin{array}{c}
\mu_{X} \\
\mu_{Y}
\end{array}\right) \text { and } \boldsymbol{\Sigma}_{\boldsymbol{W}}=\left(\begin{array}{cc}
\sigma_{X}^{2} & \rho \sigma_{X} \sigma_{Y} \\
\rho \sigma_{X} \sigma_{Y} & \sigma_{Y}^{2}
\end{array}\right),
$$

where $\rho$ is the correlation coefficient between $X$ and $Y$; $\sigma_{X}$ and $\sigma_{Y}$ are the standard deviation of $X$ and $Y$. Let $\mu_{X}$ and $\mu_{Y}$ denote their mean. Then the coefficients of variations and the standard-deviation ratio of $X$ and $Y$ are $\gamma_{X}=\frac{\sigma_{X}}{\mu_{X}} \gamma_{Y}=\frac{\sigma_{Y}}{\mu_{Y}}$ and $\omega=\frac{\sigma_{X}}{\sigma_{Y}}$, respectively.

The ratio of $X$ to $Y$ is defined as $Z=X / Y$. A number of studies on the distribution of $Z$ can be found in the literature, see, for example, Geary ${ }^{26}$, Hayya et al. ${ }^{27}$, Pham-Gia et al. ${ }^{28}$. Cedilnik et al. ${ }^{29}$ proposed 
a general explicit expression for the probability density function (p.d.f) of $Z$ while its cumulative distribution function (c.d.f) and its inverse c.d.f could be computed numerically as proposed in Celano et al. ${ }^{30}$. However, these methods are relatively complicated to be implemented. Therefore, the approximated distributions are often preferable. According to Celano and Castagliola $^{9}$, when the coefficient of variation of $X$ and $Y$ is not large, the distribution of $Z$ can be well approximated by

$$
F_{Z}\left(z \mid \gamma_{X}, \gamma_{Y}, \omega, \rho\right) \simeq \Phi\left(\frac{A}{B}\right),
$$

where $\Phi($.$) is the c.d.f of the standard normal distribution, and where A$ and $B$ are functions of $z, \gamma_{X}, \gamma_{Y}, \omega$ and $\rho$ with

$$
\begin{aligned}
A & =\frac{z}{\gamma_{Y}}-\frac{\omega}{\gamma_{X}}, \\
B & =\sqrt{\omega^{2}-2 \rho \omega z+z^{2}} .
\end{aligned}
$$

To be sure of the stability of a processes with normally distributed quality parameters, the population dispersion should be significantly smaller than the mean. Thus, the assumption about small values of $\gamma_{X}, \gamma_{Y}$ and then the approximated distribution is reasonable. Based on the c.d.f in (2), it is easy to show that the p.d.f of $Z$ is

$$
f_{Z}\left(z \mid \gamma_{X}, \gamma_{Y}, \omega, \rho\right) \simeq\left(\frac{1}{B \gamma_{Y}}-\frac{(z-\rho \omega) A}{B^{3}}\right) \times \phi\left(\frac{A}{B}\right)
$$

where $\phi($.$) is the p.d.f of the standard normal distribution. Some pictures$ of the p.d.f of $Z$ for the case $\rho=0.4, \omega=0.9 \gamma_{X} / \gamma_{Y}$ and different values of $\gamma_{X}$ and $\gamma_{Y}$ are drawn in Figure 1.

In the same way, an approximation for the i.d.f (inverse distribution function) $F_{Z}^{-1}\left(p \mid \gamma_{X}, \gamma_{Y}, \omega, \rho\right)$ of $Z$ is given by

$$
F_{Z}^{-1}\left(p \mid \gamma_{X}, \gamma_{Y}, \omega, \rho\right) \simeq \begin{cases}\frac{-C_{2}-\sqrt{C_{2}^{2}-4 C_{1} C_{3}}}{2 C_{1}} & \text { if } p \in(0,0.5], \\ \frac{-C_{2}+\sqrt{C_{2}^{2}-4 C_{1} C_{3}}}{2 C_{1}} & \text { if } p \in[0.5,1),\end{cases}
$$

where $C_{1}, C_{2}$ and $C_{3}$ are functions of $p, \gamma_{X}, \gamma_{Y}, \omega$ and $\rho$, i.e.

$$
\begin{aligned}
C_{1} & =\frac{1}{\gamma_{Y}^{2}}-\Phi^{-1}(p)^{2}, \\
C_{2} & =2 \omega\left(\rho \Phi^{-1}(p)^{2}-\frac{1}{\gamma_{X} \gamma_{Y}}\right), \\
C_{3} & =\omega^{2}\left(\frac{1}{\gamma_{X}^{2}}-\Phi^{-1}(p)^{2}\right),
\end{aligned}
$$

and where $\Phi^{-1}($.$) is the i . d . f$ of the standard normal distribution. 


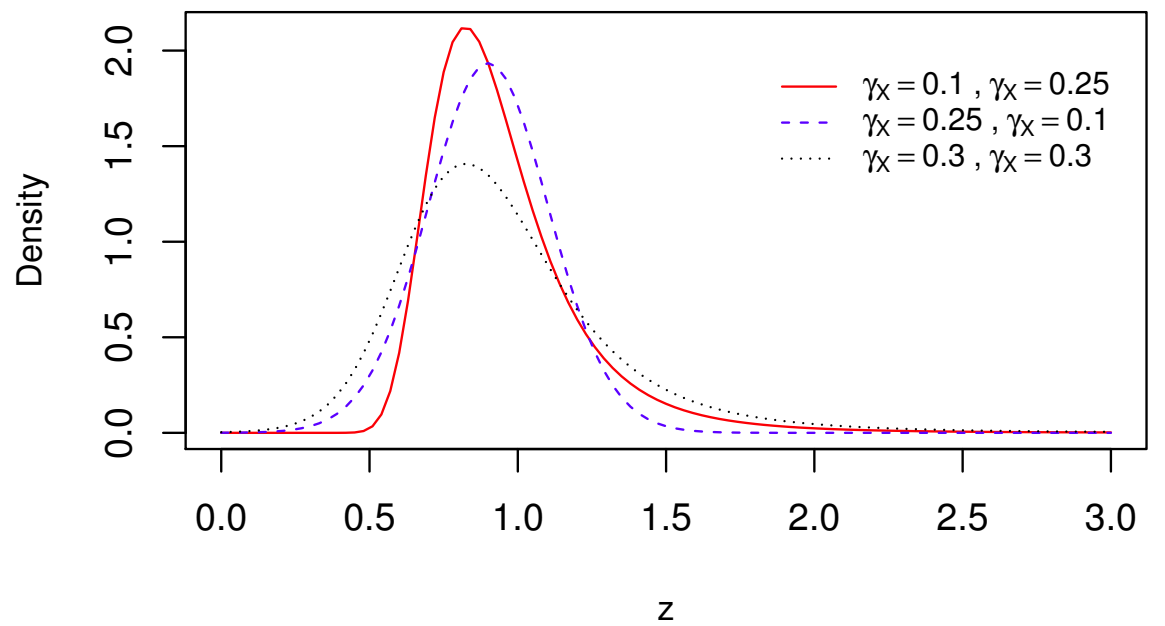

Figure 1: The probability density function of $Z$ for the case $\rho=0.4, \omega=$ $0.9 \gamma_{X} / \gamma_{Y}$ and different values of $\gamma_{X}, \gamma_{Y}$.

\section{Implementation of the one-sided Shewhart-RZ control charts}

In order to monitor the ratio $Z$, a set of $n$ samples of quality characteristic $\mathbf{W}$ at each sampling period, say $\left\{\mathbf{W}_{i, 1}, \mathbf{W}_{i, 2}, \ldots, \mathbf{W}_{i, n}\right\}$, is collected. Suppose that $\mathbf{W}_{i, 2}=\left(X_{i, j}, Y_{i, j}\right)^{T}$ is a bivariate normal vector with mean vector

$$
\boldsymbol{\mu}_{\boldsymbol{W}, \boldsymbol{i}}=\left(\begin{array}{c}
\mu_{X, i} \\
\mu_{Y, i}
\end{array}\right)
$$

and covariance matrix

$$
\boldsymbol{\Sigma}_{\boldsymbol{W}, \boldsymbol{i}}=\left(\begin{array}{cc}
\sigma_{X, i}^{2} & \rho \sigma_{X, i} \sigma_{Y, i} \\
\rho \sigma_{X, i} \sigma_{Y, i} & \sigma_{Y, i}^{2}
\end{array}\right)
$$

Similar to Celano and Castagliola ${ }^{9}$, the following assumptions are supposed:

(i) There is a linear relationship between the mean and the standard deviation of $X$ and $Y$. That is, we have $\sigma_{X, i}=\gamma_{X} \times \mu_{X, i}$ and $\sigma_{Y, i}=\gamma_{Y} \times \mu_{Y, i}$ for every $i$. Since many quality characteristics have a dispersion proportional to the population mean, it is practical to use a known and constant coefficient of variation of both $X$ and $Y$. 
(ii) when the process is in-control, the ratio $\frac{\mu_{X, i}}{\mu_{Y, i}}$ is equal to $z_{0}$ for every samples. This is an expected value to ensure the stability of the process.

(iii) the sample units are allowed to change from sample to sample, i.e. it is possible that $\boldsymbol{\mu}_{\mathbf{W}, i} \neq \boldsymbol{\mu}_{\mathbf{W}, k}$ and $\boldsymbol{\Sigma}_{\mathbf{W}, i} \neq \boldsymbol{\Sigma}_{\mathbf{W}, k}$ for $i \neq k$.

Then, the monitored statistic is

$$
\hat{Z}_{i}=\frac{\hat{\mu}_{X, i}}{\hat{\mu}_{Y, i}}=\frac{\bar{X}_{i}}{\bar{Y}_{i}}=\frac{\sum_{j=1}^{n} X_{i, j}}{\sum_{j=1}^{n} Y_{i, j}}, i=1,2, \ldots
$$

In order to determine the distribution of $\hat{Z}_{i}$, we firstly consider that $\bar{X}_{i} \sim$ $N\left(\mu_{X, i}, \frac{\sigma_{X, i}}{\sqrt{n}}\right)$ and $\bar{Y}_{i} \sim N\left(\mu_{Y, i}, \frac{\sigma_{Y, i}}{\sqrt{n}}\right)$. Then, the coefficients of variation of $\bar{X}_{i}$ and $\bar{Y}_{i}$ are

$$
\begin{gathered}
\gamma_{\bar{X}}=\frac{\mu_{X, i}}{\sigma_{X, i} \sqrt{n}}=\frac{\gamma_{X}}{\sqrt{n}}, \\
\gamma_{\bar{Y}}=\frac{\mu_{Y, i}}{\sigma_{Y, i} \sqrt{n}}=\frac{\gamma_{Y}}{\sqrt{n}} .
\end{gathered}
$$

Similarly, the standard-deviations ratio $\omega_{i}$ at each sampling period $i$ is

$$
\omega_{i}=\frac{\sigma_{X, i}}{\sigma_{Y, i}}=\frac{\mu_{X, i}}{\mu_{Y, i}} \frac{\gamma_{X}}{\gamma_{Y}}=z_{0} \times \frac{\gamma_{X}}{\gamma_{Y}}=\omega_{0} \quad \forall i=1,2, \ldots,
$$

where $\omega_{0}$ is the in-control standard-deviation ratio. The c.d.f and the $i . d . f$ of $\hat{Z}_{i}$ now can be written as

$$
\begin{gathered}
F_{\hat{Z}_{i}}\left(z \mid n, \gamma_{X}, \gamma_{Y}, z_{0}, \rho_{0}\right)=F_{Z}\left(z \mid n, \frac{\gamma_{X}}{\sqrt{n}}, \frac{\gamma_{Y}}{\sqrt{n}}, \frac{z_{0} \gamma_{X}}{\gamma_{Y}}, \rho_{0}\right) \\
F_{\hat{Z}_{i}}^{-1}\left(p \mid n, \gamma_{X}, \gamma_{Y}, z_{0}, \rho_{0}\right)=F_{Z}^{-1}\left(p \mid n, \frac{\gamma_{X}}{\sqrt{n}}, \frac{\gamma_{Y}}{\sqrt{n}}, \frac{z_{0} \gamma_{X}}{\gamma_{Y}}, \rho_{0}\right),
\end{gathered}
$$

with $F_{Z}(\ldots)$ and $F_{Z}^{-1}(\ldots)$ defined in (2) and (4).

It can be seen from the distributions in (2)-(3) (and also the Figure 1) that the distribution of $Z$ is skewed. Therefore, using a single two-sided chart to monitor both an increase and a decrease in the ratio $Z$ will lead to a $A R L$-biased chart as in Celano and Castagliola ${ }^{9}$. We overcome this problem by designing two one-sided charts monitoring the increase or the decrease of the ratio separately. Other advantages of using two one-sided charts to monitor a variable with an asymmetry distribution are further discussed in Nguyen et al. ${ }^{20}$. In particular, the two one-sided control charts consist of an upper-sided chart, which aims at detecting an increase in the ratio (denoted as Shewhort $\mathrm{PZ}^{+}$chart in the remainder of the paper), and a lower-sided chart, which aims at detecting a decrease in the ratio (denoted as Shewhart-RZ ${ }^{-}$chart in the remainder of the paper). 
Given an in-control average run length $A R L_{0}$, the lower control limit $L C L^{-}$of the Shewhart-RZ ${ }^{-}$chart is defined such that the type I error rate is equal to $\alpha_{0}$ where $A R L_{0}=1 / \alpha_{0}$. Thus, the $L C L^{-}$is equal to

$$
L C L^{-}=F_{Z}^{-1}\left(\alpha_{0} \mid \frac{\gamma_{X}}{\sqrt{n}}, \frac{\gamma_{Y}}{\sqrt{n}}, \frac{z_{0} \gamma_{X}}{\gamma_{Y}}, \rho_{0}\right) .
$$

The corresponding upper control limit $U C L^{-}$of the Shewhart-RZ ${ }^{-}$chart is $U C L^{-}=+\infty$.

Similarly, for the upward CV chart, the upper control limit $U C L^{+}$is defined such that the type I error rate is equal to $\alpha_{0}$. That means the UCL is equal to

$$
U C L^{+}=F_{Z}^{-1}\left(1-\alpha_{0} \mid \frac{\gamma_{X}}{\sqrt{n}}, \frac{\gamma_{Y}}{\sqrt{n}}, \frac{z_{0} \gamma_{X}}{\gamma_{Y}}, \rho_{0}\right),
$$

The corresponding upper control limit $L C L^{+}$of the Shewhart-RZ ${ }^{-}$chart is $L C L^{+}=-\infty$.

In the implementation of these two control charts, when $\hat{Z}<L C L^{-}$in the Shewhart-RZ ${ }^{-}$chart or when $\hat{Z}>U C L^{+}$in the Shewhart-RZ ${ }^{+}$chart, the process is considered as out-of-control, and the assignable causes should be investigated and removed. Otherwise, the process is believed to be still in-control.

Now, let us assume that the in-control ratio $z_{0}$ is shifted to $z_{1}=\tau \times z_{0}$, where $\tau>0$ is the shift size, and the in-control coefficient of correlation $\rho=\rho_{0}$ is shifted to $\rho=\rho_{1}$ because of an out-of-control condition. For the Shewhart type chart, the out-of-control average run length $A R L_{1}$ is calculated by

$$
A R L=\frac{1}{p}
$$

where $p$ is the probability of detecting a shift. In our case, the probability $p$ is definded as:

- for the Shewhart-RZ ${ }^{-}$control chart,

$$
\begin{aligned}
p & =\operatorname{Pr}\left(\hat{Z}_{i}<L C L^{-}\right) \\
& =F_{\hat{Z}}\left(L C L^{-} \mid \frac{\gamma_{X}}{\sqrt{n}}, \frac{\gamma_{Y}}{\sqrt{n}}, \frac{z_{0} \gamma_{X}}{\gamma_{Y}}, \rho_{0}\right),
\end{aligned}
$$

- for the Shewhart-RZ ${ }^{+}$control chart,

$$
\begin{aligned}
p & =\operatorname{Pr}\left(\hat{Z}_{i}<U C L^{+}\right) \\
& =1-F_{\hat{Z}}\left(U C L^{+} \mid \frac{\gamma_{X}}{\sqrt{n}}, \frac{\gamma_{Y}}{\sqrt{n}}, \frac{z_{0} \gamma_{X}}{\gamma_{Y}}, \rho_{0}\right) .
\end{aligned}
$$




\section{Performance of the one-sided Shewhart-RZ con- trol charts without the presence of measurement errors}

In this section, we investigate the performance of the one-sided Shewhart$\mathrm{RZ}$ control charts based on an assumption that there is no measurement error. For purpose of comparison, we consider the follwoing values of the process parameters, which are exactly the same those considered in ${ }^{9}$ :

- $\gamma_{X} \in\{0.01,0.2\}, \gamma_{Y} \in\{0.01,0.2\}$.

- $n \in\{1,5,7,10,15\}$.

- $\rho_{0} \in\{-0.8,-0.4,0,0.4,0.8\}$.

- $\tau \in\{0.9,0.95,0.98,0.99\}$ for the Shewhart-RZ ${ }^{-}$chart and $\tau \in\{1.01,1.02$, $1.05,1.0\}$ for the Shewhart-RZ ${ }^{+}$chart.

Also, the in-control $A R L$ is set at $A R L_{0}=200$ and the in-control ratio is $z_{0}=1$. The control limits of the one-sided Shewhart-RZ control charts for these parameters are presented in Table 1 (column titled "Without measurement error"). The out-of-control $A R L$, say $A R L_{1}$, for different values of the shift size $\tau$ corresponding to these control limits are given in Table 2 (for $\gamma_{X}=\gamma_{Y}, \rho_{0}=\rho_{1}$ ), Table $3\left(\right.$ for $\gamma_{X} \neq \gamma_{Y}, \rho_{0}=\rho_{1}$ ), Table 4 (for $\gamma_{X}=\gamma_{Y}$, $\rho_{0} \neq \rho_{1}$ ) and Table 5 (for $\gamma_{X} \neq \gamma_{Y}, \rho_{0} \neq \rho_{1}$ ). Some simple conclusions can be drawn from these tables as follows.

- In general, the control limits of the one-sided Shewhart-RZ control charts show the same behavior as those of the two-sided ShewhartRZ control chart. Given $\left(\gamma_{X}, \gamma_{Y}\right)$, the values of $L C L^{-}$and $U C L^{+}$ change with $n$ and $\rho_{0}$; and given the value of $n$ and $\rho_{0}$, the values of $L C L^{-}$and $U C L^{+}$change with $\left(\gamma_{X}, \gamma_{Y}\right)$. Moreover, the relation $L C L^{-}=1 / U C L^{+}$still holds only for the case $\gamma_{X}=\gamma_{Y}$. For instance, with $\gamma_{X}=\gamma_{Y}=0.01, \rho_{0}=0.0, n=5$, we have $L C L^{-}=0.9838=$ $1 / 1.1064=1 / U C L^{+}$(Table 1$)$.

- The one-sided Shewhart-RZ control charts overcome the $A R L$-biased problem. This is shown by the values less than $A R L_{0}=200$ of the $A R L_{1}$ in the one-sided Shewhart-RZ control chart. For example, when $\gamma_{X}=0.2, \gamma_{Y}=0.01, n=1, \rho_{1}=\rho_{0}=-0.4$ and $\tau=0.99$, we have $A R L_{1}=186.5$ for the one-sided Shewhart-RZ chart (Table 3 in this study) while $A R L_{1}=215.3>200$ for the two-sided Shewhart-RZ chart (Table 3 in Celano and Castagliola ${ }^{9}$ ).

- Besides overcoming the $A R L$-biased problem, the one-sided ShewhartRZ control charts also outperform the two-sided chart in detecting the 
process shift. For example, with $\gamma_{X}=\gamma_{Y}=0.2, \rho_{0}=\rho_{1}=0.8, n=5$ and $\tau=1.05$, we have $A R L_{1}=25.5$ in the Shewhart-RZ ${ }^{+}$chart and $A R L_{1}=43.9$ in the two-sided Shewhart-RZ chart (Table 2).

PLEASE INSERT TABLE 1 ABOUT HERE

PLEASE INSERT TABLE 2 ABOUT HERE

PLEASE INSERT TABLE 3 ABOUT HERE

PLEASE INSERT TABLE 4 ABOUT HERE

PLEASE INSERT TABLE 5 ABOUT HERE

\section{Linear covariate error model for the sample of the ratio}

In this section, we present a linear covariate error model for the sample ratio. The change of parameters of a process after being shifted under the presence of measurement error will be modeled accurately.

Consider again the bivariate normal random vector $\mathbf{W} \sim N\left(\boldsymbol{\mu}_{\mathbf{W}}, \boldsymbol{\Sigma}_{\mathbf{W}}\right)$ with the mean $\boldsymbol{\mu}_{\mathbf{W}}$ and the variance-covariacne matrix $\boldsymbol{\Sigma}_{\mathbf{W}}$ defined in (1). Let $\left\{\mathbf{W}_{i, 1}, \mathbf{W}_{i, 2}, \ldots, \mathbf{W}_{i, n}\right\}$ be a set of $n$ independent samples from this random vector where $\mathbf{W}_{i, j}=\left(X_{i, j}, Y_{i, j}\right)^{T}$ represents the quality characteristic of the $j^{\text {th }}$ sample, $j=1, \ldots, n$ at the time $i, i=1,2, \ldots$.

The measurement error means that the true quality characteristics $\mathbf{W}_{i, j}$ is unobservable. Instead, only a set of a multiple measurement from the $j^{\text {th }}$ item at the the time $i$, say $\left\{\mathbf{W}_{i, j, 1}^{*}, \mathbf{W}_{i, j, 2}^{*}, \ldots, \mathbf{W}_{i, j, m}^{*}\right\}, m \geqslant 1$, is observable. The linear covariate error model (Linna and Woodall ${ }^{31}$ ) says that these observations are related to the true value $\mathbf{W}_{i, j}$ by the following formula

$$
\mathbf{W}_{i, j, k}^{*}=\mathbf{A}+\mathbf{B W}_{i, j}+\varepsilon_{i, j, k}, k=1, \ldots, m,
$$

where $\mathbf{A}=\left(a_{X}, a_{Y}\right)^{T}$ is a $(2 \times 1)$ constatnt vector, $\mathbf{B}$ is a $(2 \times 2)$ matrix, $\varepsilon \sim N\left(\mathbf{0}, \boldsymbol{\Sigma}_{M}\right)$ is a bivariate normal random vector independent of $\mathbf{W}$. Let $\sigma_{M X}, \sigma_{M Y}$, and $\rho_{M} \in(-1,+1)$ be the standard-deviation of measurement error of $X, Y)$, and the corresponding coefficient of correlation. Then, the variance-covariance matrix $\boldsymbol{\Sigma}_{M}$ of $\varepsilon$ is denoted by

$$
\boldsymbol{\Sigma}_{M}=\left(\begin{array}{cc}
\sigma_{M X}^{2} & \rho_{M} \sigma_{M X} \sigma_{M Y} \\
\rho_{M} \sigma_{M X} \sigma_{M Y} & \sigma_{M Y}^{2}
\end{array}\right),
$$

As in Tran et al. ${ }^{8}$, we consider the case $\mathbf{B}=\mathbf{I}_{2 \times 2}$, an identity matrix. That is a constant vector of accuracy error $\mathbf{A}$ within the range of measurements is assumed. 
Since the true value of $\mathbf{W}_{i, j}$ is not observed, the mean

$$
\overline{\mathbf{W}}_{i, j}^{*}=\frac{1}{m} \sum_{k=1}^{m} \mathbf{W}_{i, j, k}^{*}=\mathbf{A}+\mathbf{B} \mathbf{W}_{i, j}+\frac{1}{m} \sum_{k=1}^{m} \varepsilon_{i, j, k}
$$

is often considered as its representation. $\overline{\mathbf{W}}_{i, j}^{*}=\left(\bar{X}_{i, j}^{*}, \bar{Y}_{i, j}^{*}\right)$ of the observable quantities $\left\{\mathbf{W}_{i, j, 1}^{*}, \mathbf{W}_{i, j, 2}^{*}, \ldots, \mathbf{W}_{i, j, m}^{*}\right\}$ is often considered as a represented value for the true value $\mathbf{W}_{i, j}$. Formula (20) shows that $\overline{\mathbf{W}}_{i, j}^{*}$ is also a bivariate normal random vector with the mean vector

$$
\mu_{\mathbf{W}^{*}}=\mathbf{A}+\mathbf{B} \mu_{W}
$$

and the variance-covariance matrix

$$
\boldsymbol{\Sigma}_{\mathbf{W}^{*}}=\mathbf{B} \boldsymbol{\Sigma}_{\boldsymbol{W}} \mathbf{B}^{T}+\boldsymbol{\Sigma}_{M}=\boldsymbol{\Sigma}_{\boldsymbol{W}}+\frac{1}{m} \boldsymbol{\Sigma}_{M} .
$$

Based on these general formulas, in the sequel we will study the change of the process parameters under the measurement error when the process is shifted.

When the process is in-control, we denote the mean vector of the true value $\mathbf{W}_{i, j}=\left(X_{i, j}, Y_{i, j}\right)$ as $\boldsymbol{\mu}_{\mathbf{0}, \boldsymbol{W}}=\left(\mu_{0, X}, \mu_{0, Y}\right)^{T}$ and the coefficient of correlation between $X_{i, j}$ and $Y_{i, j}$ as $\rho=\rho_{0}$. As a result, the mean ratio is $z_{0}=\frac{\mu_{0, X}}{\mu_{0, Y}}$. Under an abnormal condition, suppose that the in-control ratio $z_{0}$ is shifted to $z_{1}=\tau z_{0}$, where $\tau$ is the shift size, and the in-control coefficient of correlation $\rho=\rho_{0}$ is shifted to $\rho=\rho_{1}$. The shift of $z_{0}$ can be expressed by the shift of $\boldsymbol{\mu}_{\boldsymbol{W}}$ from the in-control value $\boldsymbol{\mu}_{\mathbf{0}, \boldsymbol{W}}$ to the outof-control value $\boldsymbol{\mu}_{\mathbf{1}, \boldsymbol{W}}=\left(\mu_{0, X}+\delta_{X} \sigma_{X}, \mu_{0, Y}+\delta_{Y} \sigma_{Y}\right)^{T}$, where $\delta_{X}$ and $\delta_{Y}$ represent the size of the mean shift of $X_{i, j}$ and $Y_{i, j}$, the standard deviation $\sigma_{X}$ and $\sigma_{Y}$ are supposed to be the same. The out-of-control mean ratio is now equal to

$$
z_{1}=\frac{\mu_{0, X}+\delta_{X} \sigma_{X}}{\mu_{0, Y}+\delta_{Y} \sigma_{Y}}=\tau \times z_{0}=\tau \times \frac{\mu_{0, X}}{\mu_{0, Y}},
$$

Thus, the shift size $\tau$ can be expressed as

$$
\tau=\frac{1+\delta_{X} \gamma_{X}}{1+\delta_{Y} \gamma_{Y}}
$$

or equivalent,

$$
1+\delta_{X} \gamma_{X}=\tau\left(1+\delta_{Y} \gamma_{Y}\right) .
$$

Now, if we express the mean vector and the variance-covariance matrix of $\overline{\mathbf{W}}_{i, j}^{*}$ in the form

$$
\boldsymbol{\mu}_{\boldsymbol{W}^{*}}=\left(\begin{array}{c}
\mu_{X^{*}} \\
\mu_{Y^{*}}
\end{array}\right) \text { and } \boldsymbol{\Sigma}_{\boldsymbol{W}^{*}}=\left(\begin{array}{cc}
\sigma_{X^{*}}^{2} & \rho^{*} \sigma_{X} \sigma_{Y} \\
\rho^{*} \sigma_{X^{*}} \sigma_{Y^{*}} & \sigma_{Y^{*}}^{2}
\end{array}\right),
$$


the equations (21) and (22) lead to

$$
\begin{aligned}
\mu_{X^{*}} & =a_{X}+\mu_{X}+\delta_{X} \sigma_{X}, \\
\mu_{Y^{*}} & =a_{Y}+\mu_{Y}+\delta_{Y} \sigma_{Y}, \\
\sigma_{X^{*}}^{2} & =\sigma_{X}^{2}+\frac{\sigma_{M X}^{2}}{m}, \\
\sigma_{Y^{*}}^{2} & =\sigma_{Y}^{2}+\frac{\sigma_{M Y}^{2}}{m}, \\
\rho^{*} & =\frac{\rho \sigma_{X} \sigma_{Y}+\rho_{M} \frac{\sigma_{M X} \sigma_{M Y}}{m}}{\sigma_{X^{*}} \sigma_{Y^{*}}} .
\end{aligned}
$$

Therefore, the coefficients of variation $\gamma_{X^{*}}=\frac{\sigma_{X^{*}}}{\mu_{X^{*}}}$ and $\gamma_{Y^{*}}=\frac{\sigma_{Y^{*}}}{\mu_{Y^{*}}}$ of $\bar{X}_{i, j}^{*}$ and $\bar{Y}_{i, j}^{*}$ are equal to

$$
\begin{aligned}
\gamma_{X^{*}} & =\frac{\sqrt{\sigma_{X}^{2}+\frac{\sigma_{M X}^{2}}{m}}}{a_{X}+\mu_{0, X}+\delta_{X} \sigma_{X}}, \\
\gamma_{Y^{*}} & =\frac{\sqrt{\sigma_{Y}^{2}+\frac{\sigma_{M Y}^{2}}{m}}}{a_{Y}+\mu_{0, Y}+\delta_{X} \sigma_{Y}} .
\end{aligned}
$$

If we divide the numerator of the fraction in (30) by $\sigma_{X}$, its denominator by $\mu_{0, X}$ and then replace $1+\delta_{X} \sigma_{X}$ by $\tau\left(1+\delta_{Y} \sigma_{Y}\right)$ as in (23), the coefficient of variation $\gamma_{X^{*}}$ in (30) can be rewritten as

$$
\gamma_{X^{*}}=\frac{\sqrt{1+\frac{\eta_{X}^{2}}{m}}}{1+\delta_{X} \gamma_{X}+\theta_{X}} \times \gamma_{X}=\frac{\sqrt{1+\frac{\eta_{X}^{2}}{m}}}{\tau\left(1+\delta_{Y} \gamma_{Y}\right)+\theta_{X}} \times \gamma_{X}
$$

where $\eta_{X}=\frac{\sigma_{M X}}{\sigma_{X}}, \theta_{X}=\frac{a_{X}}{\mu_{0, X}}$, and $\gamma_{X}=\frac{\sigma_{X}}{\mu_{0, X}}$.

Similarly, the coefficients of variation $\gamma_{Y^{*}}$ in (31) and the coefficient of correlation $\rho^{*}$ in (29) can be expressed by

$$
\begin{aligned}
\gamma_{Y^{*}} & =\frac{\sqrt{1+\frac{\eta_{Y}^{2}}{m}}}{1+\delta_{Y} \gamma_{Y}+\theta_{Y}} \times \gamma_{Y}, \\
\rho^{*} & =\frac{\rho+\rho_{M} \frac{\eta_{X} \eta_{Y}}{m}}{\sqrt{1+\eta_{X}^{2} / m} \sqrt{1+\eta_{Y}^{2} / m}},
\end{aligned}
$$

where $\eta_{Y}=\frac{\sigma_{M Y}}{\sigma_{Y}}, \theta_{Y}=\frac{a_{Y}}{\mu_{0, Y}}$ and $\gamma_{Y}=\frac{\sigma_{Y}}{\mu_{0, Y}}$. In addition, the standarddeviation ratio $\omega^{*}=\frac{\sigma_{X^{*}}}{\sigma_{Y^{*}}}$ is equal to

$$
\omega^{*}=\sqrt{\frac{1+\frac{\eta_{X}^{2}}{m}}{1+\frac{\eta_{Y}^{2}}{m}}} \times \omega
$$


where $\omega=\frac{\sigma_{X}}{\sigma_{Y}}$. By these notations, it can be seen that the in-control and out-of-control ratio under the presence of the measurement error are

$$
\begin{aligned}
& z_{0}^{*}=\frac{\mu_{0, X^{*}}}{\mu_{0, Y^{*}}}=\frac{\mu_{0, X}+a_{X}}{\mu_{0, Y}+a_{Y}}=\frac{1+\theta_{X}}{1+\theta_{Y}} \times z_{0} \\
& z_{1}^{*}=\frac{\mu_{1, X^{*}}}{\mu_{1, Y^{*}}}=\frac{a_{X}+\mu_{0, X}+\delta_{X} \sigma_{X}}{a_{Y}+\mu_{0, Y}+\delta_{Y} \sigma_{Y}}=\frac{1+\theta_{X}+\delta_{X} \gamma_{X}}{1+\theta_{Y}+\delta_{Y} \gamma_{Y}} \times z_{0}
\end{aligned}
$$

The equations (36)-(37) show that in general we do not have $z_{1}^{*}=\tau z_{0}^{*}$.

\section{Design and implementation of the Shewhart-RZ control chart with measurement error}

Under the presence of measurement error, the monitored statistic in (7) is of the form

$$
\hat{Z}_{i}^{*}=\frac{\hat{\mu}_{X_{i}^{*}}}{\hat{\mu}_{Y_{i}^{*}}}=\frac{\bar{X}_{i}^{*}}{\bar{Y}_{i}^{*}}=\frac{\sum_{j=1}^{n} X_{i, j}^{*}}{\sum_{j=1}^{n} Y_{i, j}^{*}} .
$$

where $\overline{\bar{X}}_{i}^{*}=\frac{1}{n} \sum_{j=1}^{n} \bar{X}_{i, j}^{*}, \overline{\bar{Y}}_{i}^{*}=\frac{1}{n} \sum_{j=1}^{n} \bar{Y}_{i, j}^{*}, \bar{X}_{i, j}^{*}$ and $\bar{Y}_{i, j}^{*}$ are two components of the bivariate normal vector $\overline{\mathbf{W}}_{i, j}^{*}$ in (20).

From their definition, it is easy to see that $\overline{\bar{X}}_{i}^{*} \sim N\left(\mu_{X^{*}}, \frac{\sigma_{X^{*}}}{\sqrt{n}}\right)$ and $\overline{\bar{X}}_{i}^{*} \sim N\left(\mu_{Y^{*}}, \frac{\sigma_{Y^{*}}}{\sqrt{n}}\right)$, the coefficients of variation of $\overline{\bar{X}}_{i}^{*}$ and $\overline{\bar{Y}}_{i}^{*}$ are now

$$
\begin{aligned}
& \gamma_{\bar{X}^{*}}=\frac{\sigma_{X^{*}}}{\mu_{X^{*} \sqrt{n}}}=\frac{\gamma_{X^{*}}}{\sqrt{n}}, \\
& \gamma_{\bar{Y}^{*}}=\frac{\sigma_{Y^{*}}}{\mu_{Y^{*} \sqrt{n}}}=\frac{\gamma_{Y^{*}}}{\sqrt{n}},
\end{aligned}
$$

and the standard-deviation ratio $\omega_{i}^{*}$ of $\overline{\bar{X}}_{i}^{*}$ and $\overline{\bar{Y}}_{i}^{*}$ is

$$
\omega_{i}^{*}=\frac{\sigma_{X^{*}} / \sqrt{n}}{\sigma_{Y^{*}} / \sqrt{n}}=\frac{\sigma_{X^{*}}}{\sigma_{Y^{*}}}=\omega^{*}
$$

The c.d.f and the inverse c.d.f of $\hat{Z}_{i}^{*}$ now can be obtained from (11) and (12) where $\gamma_{X}, \gamma_{Y}, \omega$ and $\rho$ are replaced by $\gamma_{\bar{X}^{*}}, \gamma_{\bar{Y}^{*}}, \omega^{*}$ and $\rho^{*}$ defined in (39), (40), (41) and (34).

The design and the implementation of the one-sided Shewhart-RZ control chart under the presence of measurement error are similar to those of the one-sided Shewhart-RZ control chart without measurement error. The different is that the distribution of $\hat{Z}_{i}$ in (13)-(14) and (16)-(17) should be replaced by the distribution of $\hat{Z}_{i}^{*}$.

In the previous section, we evaluate the performance of the Shewhart$\mathrm{RZ}$ control chart through the $A R L$. This measurement is calculated based on a predetermined specific shift size. However, it is no doubt that specific 
size of the shift in the ratio can not be predetermined exactly. Therefore, in practice, quality practitioners prefer to consider a range of the shift size, say $\Omega=[a, b]$ rather than a particular size. Moreover, the use of a uniform distribution has been widely suggested to represent the unknown shift sizes in the literature. Following this argument, we now evaluate the performance of the proposed charts in the presence of measurement error through the $E A R L$ (expected average run length), which is defined as

$$
E A R L=\int_{\Omega} A R L \times f_{\tau}(\tau) \mathrm{d} \tau
$$

where $f_{\tau}(\tau)=\frac{1}{b-a}$ for $\tau \in \Omega$. Similar to Tran et al. ${ }^{8}$, the following two different ranges of the shift size are considered: $\Omega_{D}=[0.9,1)$ for a decreasing case of $\tau$ and $\Omega_{I}=[1,1.1)$ for an increasing case of $\tau$.

\section{The effect of measurement error on the one- sided Shewhart-RZ control charts}

The effect of measurement error on the proposed charts is presented in this section. Without loss of generality, the in-control values of the ratio and the $A R L$ are supposed to be $z_{0}=1$ and $A R L_{0}=200$. We also suppose that the size of the mean shift of $Y_{i, j}$ is $\delta_{Y}=1$. Moreover, the values of $\gamma_{X}, \gamma_{Y}$ and $\rho_{0}$ are considered the same as those in the section 4, except for the case of $n$ : we consider only two values of the sample size, $n \in\{1,15\}$, to avoid the cumbersome in presented results.

Table 1 (the rightmost block titled "with measurement error") shows the control limits $L C L^{-}$and $U C L^{+}$of the one-sided Shewhart-RZ control charts in the presence of measurement error for the case $\theta_{X}=\theta_{Y}=0.01$, $\eta_{X}=\eta_{Y}=0.01$ and $\rho_{M}=0.5$. As can be seen from this table, the measurement error affects the value of the control limits. However, the behavior of the control limits in the presence of the measurement error is still the same as that in the case of no measurement error. For example, we still have $U C L^{+}=1 / L C L^{-}$when $\gamma_{X}=\gamma_{Y}$.

In order to see the effect of precision errors (represented by $\eta_{X}$ and $\eta_{Y}$ ) on the performance of the one-sided Shewhart-RZ charts, we fix the value of $\gamma_{X}=\gamma_{Y} \in\{0.01,0.2\}, \rho_{M}=0, \theta_{X}=\theta_{Y}=0, \rho_{0}=\rho_{1}=-0.8$ or $\rho_{0}=-0.4, \rho_{1}=-0.8$, and we considere a range of $\eta_{X}$ and $\eta_{Y}$, which is $\eta_{X} \in\{0,0.1,0.2, \ldots, 1\}$ and $\eta_{Y} \in\{0,0.1,0.2, \ldots, 1\}$. The corresponding $E A R L$ values are presented in Figures 2-3. Since the increase of $\eta_{X}$ and $\eta_{Y}$ leads to the increase of the $E A R L$, we can say that the precision errors have a negative impact on the charts' performance. For example, given $n=$ $1, \Omega_{D}=(1,1.1], \rho_{0}=\rho_{1}=0.8, \gamma_{X}=\gamma_{Y}=0.2$, we obtain $E A R L=151.93$ when $\eta_{X}=\eta_{Y}=0.1$ and $E A R L=168.85$ when $\eta_{X}=\eta_{Y}=1$ (Figure 2). However, this influence is negligible when the precision errors are not too 
large. For instance, in Figure 3 with $n=1, \Omega_{I}=[0.9,1), \rho_{0}=0.4, \rho_{1}=0.8$, $\gamma_{X}=\gamma_{Y}=0.01$, we have $E A R L=5.23$ when $\eta_{X}=\eta_{Y}=0.5$ compared to $E A R L=4.85$ when $\eta_{X}=\eta_{Y}=0.0$, which corresponds to the case of no measurement error.

\section{PLEASE INSERT FIGURE 2 ABOUT HERE PLEASE INSERT FIGURE 3 ABOUT HERE}

We investigate the effect of accuracy errors (represented by $\theta_{X}$ and $\theta_{Y}$ ) on the global performance of the Shewhart-RZ control chart in Figures 4-5 by allowing $\theta_{X}$ and $\theta_{Y}$ to take the value in $\{0,0.005,0.01, \ldots, 0.05\}$ and fixing $\gamma_{X}=\gamma_{Y} \in\{0.01,0.2\}, \rho_{M}=0, \eta_{X}=\eta_{Y}=0, \rho_{0}=\rho_{1}=-0.8$ (Figure 2) and $\rho_{0}=-0.4, \rho_{1}=-0.8$ (Figure 3). These figures show that the effect of $\theta_{X}$ and $\theta_{Y}$ on the value of $E A R L$ depends on the value of $\rho_{0}$ and $\rho_{1}$. In this study, when $\rho_{0}=\rho_{1}=-0.8$, the increase of $\theta_{X}$ and the decrease of $\theta_{Y}$ lead to the increase of the $E A R L$ (Figure 2). In contrast, when $\rho_{0}=-0.4, \rho_{1}=-0.8$, the EARL increases when $\theta_{X}$ decreases and $\theta_{Y}$ increases. Take the case $n=1, \Omega_{I}=[0.9,1), \rho_{0}=\rho_{1}=0.8, \gamma_{X}=\gamma_{Y}=0.2$ in Figure 4 as an example: we have $E A R L=104.60$ when $\theta_{X}=0.005, \theta_{Y}=0.05$ and $E A R L=113.56$ when $\theta_{X}=0.05, \theta_{Y}=0.005$. Moreover, it is also important to see that the larger value of $n$ improves significantly the charts' performance: the values of $E A R L$ corresponding to $n=15$ are always smaller than those corresponding to $n=1$ regardless of the presence of measurement error.

\section{PLEASE INSERT FIGURE 4 ABOUT HERE PLEASE INSERT FIGURE 5 ABOUT HERE}

The effect of $\rho_{M}$ on the one-sided Shewhart-RZ control charts is illustrated in Figure 6 (for $\rho_{0}=\rho_{1}=-0.8$ ) and Figure 7 (for $\rho_{0}=-0.4, \rho_{1}=$ $-0.8)$, where we fix $\gamma_{X}=\gamma_{Y} \in\{0.01,0.2\}, \theta_{X}=\theta_{Y}=0.05, \eta_{X}=\eta_{Y}=0.28$ and we let $\rho_{M}$ to take the values in $\{-1,-0.9, \ldots, 0.9,1\}$. As can be seen from these figures, the larger the value of $\rho_{M}$ is, the smaller the value of $E A R L$ is. For example, with $n=1, \Omega_{D}=[0.9,1), \rho_{0}=\rho_{1}=-0.8, \gamma_{X}=$ $\gamma_{Y}=0.2$, we have $E A R L=58.60$ when $\rho_{M}=-0.8$ and $E A R L=56.19$ when $\rho_{M}=0.8$ (Figure 7 ).

\section{PLEASE INSERT FIGURE 6 ABOUT HERE PLEASE INSERT FIGURE 7 ABOUT HERE}

It is recommended in several studies in the literature that take multiple measurements per item is a way to compensate for the negative effect of measurement error on the performance of a control chart. In order to check if this property is still the case for the Shewhart-RZ control chart, we calculate the value of $E A R L$ when $m$ increases from 1 to 10 given the values of $\gamma_{X}=\gamma_{Y} \in\{0.01,0.2\}, \theta_{X}=\theta_{Y}=0.05$, and $\eta_{X}=\eta_{Y}=0.28$. 
The obtained results are presented in Figures 8-9. These results show a different conclusion that the increase of $m$ does not improve significantly the Shewhart-RZ charts' performance. For example, with $n=1, \Omega_{D}=[0.9,1)$, $\gamma_{X}=\gamma_{Y}=0.2, \theta_{X}=\theta_{Y}=0.05, \eta_{X}=\eta_{Y}=0.28$ and $\rho_{0}=-0.4, \rho_{1}=-0.8$ in Figure 9, we obtain an insignificant decrease from $E A R L=58.54$ when $m=1$ to $E A R L=58.18$ when $m=10$.

\section{PLEASE INSERT FIGURE 8 ABOUT HERE PLEASE INSERT FIGURE 9 ABOUT HERE}

\section{Illustrative example}

In this section, we present an illustrative example of the implementation of the one-sided Shewhart-RZ control chart in the presence of the measurement error. We consider a real situation of managing waste batteries in some battery recycling plants in Italy, which was introduced in Tran et al. ${ }^{8}$.

At the first step of the recycling process, the batteries are received by collecting facilities incoming disposed material into designated drums, sacks or boxes, denoted as "batches". Many disposed materials that are not recyclable batteries like small electronic devices, metals and other kinds of waste might be included in these batches. To reduce the recycling cost, removing these disposed materials from the batches is required. In a recycling plant, the ratio $Z$ of recyclable batteries weight (denoted by $X$ ) to total batch weight (denoted by $Y$ ) is monitored to quantify the cost. Similar to Tran et al. ${ }^{8}$, we suppose that a value of 0.95 is the value of interest for the in-control ratio $z_{0}$ to avoid an economic loss.

At regular intervals $i=1,2, \ldots$, a sample of $n=5$ is collected with batches having nominal weight $100 \mathrm{~kg}$ in the process of monitoring the ratio $Z$. Because of the variability, the batch weight is considered as a random normal variable $Y \sim N(100,1)$. The recyclable batteries' weight within each batch is also considered as a normal random variable with a mean of $\mu_{X}=$ $95 \mathrm{~kg}$. The sample average weights $\bar{X}_{i}^{*}=\frac{1}{n} \sum_{j=1}^{n} X_{i, j}^{*}$ and $\bar{Y}_{i}^{*}=\frac{1}{n} \sum_{j=1}^{n} Y_{i, j}^{*}$ are recorded. a set of simulated samples of incoming material in the battery recycling process introduced in Tran et al. ${ }^{8}$ has been reproduced in Table 6. A decreasing shift has been simulated from sample \#11 with the size up to $1 \%$ of the in-control ratio $z_{0}$. Moreover, the coefficients of variations of two variables are $\gamma_{X}=0.01$ and $\gamma_{Y}=0.01$, and the in-control correlation coefficient between them is $\rho_{0}=0.8$.

We suppose the following parameters of the linear covariate error model: $\theta_{X}=\theta_{Y}=0, \eta_{X}=\eta_{Y}=0.28, \rho_{1}=0.8$ and $\rho_{M}=0$. Also, we apply a smoothing value of $\lambda=0.2$. Then, the control limit of the Shewhart-RZ ${ }^{-}$ control chart with measurement errors is $L C L^{-}=0.9419487$.

The Shewhart- $\mathrm{RZ}^{-}$control chart corresponding to this experiment is illustrated in Figure 10. As can be seen from this figure, the samples \#11 is 
plotted below the control limit, indicating that an assignable cause may have appeared to shift the process to the out-of-control condition. This result is in accord with the finding in Tran et al. ${ }^{8}$.

PLEASE INSERT TABLE 6 ABOUT HERE

PLEASE INSERT FIGURE 10 ABOUT HERE

\section{Concluding remarks}

In this paper, we have proposed two one-sided Shewhart control charts to monitor the ratio between two random normal variables. This is to overcome the $A R L$-biased problem of the two-sided Shewhart-RZ control chart designed in Celano and Castagliola ${ }^{9}$. These one-sided charts also outperform the two-sided Shewhart-RZ control chart in detecting process shifts. Moreover, we have modeled the change of parameters when the process is shifted from an in-control condition to an out-of-control status under the presence of measurement error in detail. The negative effect of measurement errors on the performance of the two one-sided Shewhart-RZ charts has been also explored. Reducing these negative effects on the ratio control chart by combining with adaptive strategies like variable sampling interval or with run rules could be an interest in future research.

\section{References}

[1] H. Ren, N. Chen and Z. Wang. Phase-II monitoring in multichannel profile observations. Journal of Quality Technology, 51(4):338-352, 2018.

[2] A. Mukherjee and A. C. Rakitzis. Some simultaneous progressive monitoring schemes for the two parameters of a zero-inflated Poisson process under unknown shifts. Journal of Quality Technology, 51(3):257-283, 2019 .

[3] L.L. Ho, F.H. Fernandes and M. Bourguignon. Control charts to monitor rates and proportions. Quality and Reliability Engineering International, 35(1):74-83, 2019.

[4] H.D. Nguyen, K.P. Tran and C. Heuchenne. Monitoring the ratio of two normal variables using variable sampling interval exponentially weighted moving average control charts. Quality and Reliability Engineering International, 35(1):439-460, 2018.

[5] W.A. Shewhart. Economic control of quality of manufactured product. Van Nostrand, New York, 1931. 
Table 1: Values of $L C L^{-}$(first row) and $U C L^{+}$(second row) for the one-sided Shewhart-RZ control charts in the presence of Measurement Error, for $z_{0}=1, A R L_{0}=200, \theta_{X}=\theta_{Y}=0.01, \eta_{X}=\eta_{Y}=0.28$, $\rho_{M}=0.5, n \in\{1,5,7,10,15\}, \gamma_{X} \in\{0.01,0.2\}, \gamma_{Y} \in\{0.01,0.2\}$ and $\rho_{0} \in\{-0.8,-0.4,0,0.4,0.8\}$

\begin{tabular}{|c|c|c|c|c|c|c|c|c|c|c|c|c|}
\hline & \multirow[b]{2}{*}{$\gamma_{Y}$} & \multirow[b]{2}{*}{$\rho_{0}$} & \multicolumn{5}{|c|}{ Without measurement error } & \multicolumn{5}{|c|}{ With measurement error } \\
\hline & & & $n=1$ & $n=5$ & $n=7$ & $n=10$ & $n=15$ & $n=1$ & $n=5$ & $n=7$ & $n=10$ & $n=15$ \\
\hline \multirow[t]{10}{*}{0.01} & 0.01 & -0.8 & 0.9523 & 0.9784 & 0.9817 & 0.9847 & 0.9875 & 0.9527 & 0.9786 & 0.9819 & 0.9848 & 0.9876 \\
\hline & & & 1.0501 & 1.0221 & 1.0186 & 1.0156 & 1.0127 & 1.0496 & 1.0219 & 1.0185 & 1.0154 & 1.0126 \\
\hline & & -0.4 & 0.9578 & 0.9809 & 0.9838 & 0.9865 & 0.9889 & 0.9580 & 0.9810 & 0.9839 & 0.9865 & 0.9890 \\
\hline & & & 1.0441 & 1.0195 & 1.0164 & 1.0137 & 1.0112 & 1.0438 & 1.0193 & 1.0163 & 1.0136 & 1.0111 \\
\hline & & 0.0 & 0.9642 & 0.9838 & 0.9863 & 0.9885 & 0.9906 & 0.9642 & 0.9838 & 0.9863 & 0.9886 & 0.9906 \\
\hline & & & 1.0371 & 1.0164 & 1.0139 & 1.0116 & 1.0095 & 1.0371 & 1.0164 & 1.0139 & 1.0116 & 1.0094 \\
\hline & & 0.4 & 0.9722 & 0.9875 & 0.9894 & 0.9911 & 0.9927 & 0.9718 & 0.9873 & 0.9893 & 0.9910 & 0.9927 \\
\hline & & & 1.0286 & 1.0127 & 1.0107 & 1.0090 & 1.0073 & 1.0290 & 1.0129 & 1.0109 & 1.0091 & 1.0074 \\
\hline & & 0.8 & 0.9838 & 0.9927 & 0.9939 & 0.9949 & 0.9958 & 0.9827 & 0.9922 & 0.9934 & 0.9945 & 0.9955 \\
\hline & & & 1.0164 & 1.0073 & 1.0062 & 1.0052 & 1.0042 & 1.0176 & 1.0078 & 1.0066 & 1.0055 & 1.0045 \\
\hline \multirow[t]{10}{*}{0.20} & 0.20 & -0.8 & 0.3375 & 0.6405 & 0.6877 & 0.7320 & 0.7757 & 0.4141 & 0.6904 & 0.7321 & 0.7710 & 0.8091 \\
\hline & & & 2.9631 & 1.5613 & 1.4542 & 1.3662 & 1.2891 & 2.4147 & 1.4483 & 1.3658 & 1.2970 & 1.2359 \\
\hline & & -0.4 & 0.3800 & 0.6746 & 0.7184 & 0.7593 & 0.7992 & 0.4561 & 0.7203 & 0.7588 & 0.7944 & 0.8291 \\
\hline & & & 2.6316 & 1.4823 & 1.3919 & 1.3171 & 1.2512 & 2.1924 & 1.3883 & 1.3179 & 1.2588 & 1.2062 \\
\hline & & 0.0 & 0.4377 & 0.7166 & 0.7559 & 0.7922 & 0.8274 & 0.5109 & 0.7564 & 0.7908 & 0.8222 & 0.8527 \\
\hline & & & 2.2849 & 1.3955 & 1.3229 & 1.2624 & 1.2087 & 1.9574 & 1.3220 & 1.2646 & 1.2162 & 1.1728 \\
\hline & & 0.4 & 0.5236 & 0.7721 & 0.8049 & 0.8347 & 0.8634 & 0.5883 & 0.8032 & 0.8317 & 0.8576 & 0.8825 \\
\hline & & & 1.9100 & 1.2952 & 1.2424 & 1.1980 & 1.1582 & 1.6997 & 1.2451 & 1.2024 & 1.1660 & 1.1332 \\
\hline & & 0.8 & 0.6853 & 0.8611 & 0.8821 & 0.9009 & 0.9186 & 0.7212 & 0.8744 & 0.8933 & 0.9103 & 0.9263 \\
\hline & & & 1.4592 & 1.1614 & 1.1337 & 1.1100 & 1.0886 & 1.3866 & 1.1437 & 1.1194 & 1.0986 & 1.0795 \\
\hline \multirow[t]{10}{*}{0.01} & 0.20 & -0.8 & 0.6462 & 0.8052 & 0.8304 & 0.8542 & 0.8778 & 0.6823 & 0.8290 & 0.8517 & 0.8729 & 0.8939 \\
\hline & & & 2.1053 & 1.3114 & 1.2516 & 1.2025 & 1.1596 & 1.8208 & 1.2553 & 1.2078 & 1.1684 & 1.1335 \\
\hline & & -0.4 & 0.6527 & 0.8088 & 0.8336 & 0.8569 & 0.8801 & 0.6878 & 0.8319 & 0.8542 & 0.8751 & 0.8957 \\
\hline & & & 2.0843 & 1.3056 & 1.2468 & 1.1987 & 1.1566 & 1.8063 & 1.2508 & 1.2042 & 1.1654 & 1.1311 \\
\hline & & 0.0 & 0.6594 & 0.8125 & 0.8368 & 0.8597 & 0.8824 & 0.6934 & 0.8350 & 0.8569 & 0.8774 & 0.8976 \\
\hline & & & 2.0632 & 1.2996 & 1.2420 & 1.1948 & 1.1536 & 1.7916 & 1.2463 & 1.2005 & 1.1624 & 1.1288 \\
\hline & & 0.4 & 0.6662 & 0.8162 & 0.8401 & 0.8625 & 0.8848 & 0.6992 & 0.8381 & 0.8596 & 0.8797 & 0.8995 \\
\hline & & & 2.0419 & 1.2936 & 1.2372 & 1.1909 & 1.1505 & 1.7769 & 1.2417 & 1.1967 & 1.1594 & 1.1264 \\
\hline & & 0.8 & 0.6734 & 0.8201 & 0.8434 & 0.8654 & 0.8872 & 0.7051 & 0.8412 & 0.8623 & 0.8820 & 0.9015 \\
\hline & & & 2.0203 & 1.2875 & 1.2322 & 1.1869 & 1.1473 & 1.7619 & 1.2370 & 1.1929 & 1.1563 & 1.1239 \\
\hline \multirow[t]{10}{*}{0.20} & 0.01 & -0.8 & 0.4750 & 0.7625 & 0.7990 & 0.8316 & 0.8624 & 0.4668 & 0.7591 & 0.7961 & 0.8292 & 0.8604 \\
\hline & & & 1.5476 & 1.2420 & 1.2042 & 1.1706 & 1.1392 & 1.5540 & 1.2451 & 1.2069 & 1.1729 & 1.1410 \\
\hline & & -0.4 & 0.4798 & 0.7659 & 0.8020 & 0.8343 & 0.8646 & 0.4712 & 0.7623 & 0.7990 & 0.8317 & 0.8625 \\
\hline & & & 1.5322 & 1.2364 & 1.1997 & 1.1669 & 1.1362 & 1.5393 & 1.2398 & 1.2025 & 1.1693 & 1.1382 \\
\hline & & 0.0 & 0.4847 & 0.7694 & 0.8051 & 0.8369 & 0.8669 & 0.4758 & 0.7656 & 0.8019 & 0.8342 & 0.8647 \\
\hline & & & 1.5166 & 1.2308 & 1.1951 & 1.1632 & 1.1332 & 1.5246 & 1.2345 & 1.1982 & 1.1658 & 1.1354 \\
\hline & & 0.4 & 0.4897 & 0.7730 & 0.8083 & 0.8397 & 0.8692 & 0.4805 & 0.7690 & 0.8049 & 0.8368 & 0.8669 \\
\hline & & & 1.5010 & 1.2251 & 1.1904 & 1.1594 & 1.1302 & 1.5097 & 1.2291 & 1.1937 & 1.1622 & 1.1325 \\
\hline & & 0.8 & 0.4950 & 0.7767 & 0.8115 & 0.8425 & 0.8716 & 0.4854 & 0.7724 & 0.8079 & 0.8395 & 0.8691 \\
\hline & & & 1.4851 & 1.2193 & 1.1856 & 1.1555 & 1.1271 & 1.4946 & 1.2236 & 1.1892 & 1.1585 & 1.1296 \\
\hline
\end{tabular}


Table 2: $A R L$ of the one-sided Shewhart-RZ control charts for $\gamma_{X} \in\{0.01,0.2\}, \gamma_{Y} \in$ $\{0.01,0.2\}, \gamma_{X}=\gamma_{Y}, \rho_{0} \in\{-0.8,-0.4,0,0.4,0.8\}, \rho_{0}=\rho_{1}, n \in\{1,5,7,10,15\}$.

\begin{tabular}{|c|c|c|c|c|c|c|c|c|c|c|}
\hline \multirow[b]{2}{*}{$\tau$} & \multicolumn{5}{|c|}{$\left(\gamma_{X}=0.01, \gamma_{Y}=0.01\right)$} & \multicolumn{5}{|c|}{$\left(\gamma_{X}=0.2, \gamma_{Y}=0.2\right)$} \\
\hline & $n=1$ & $n=5$ & $n=7$ & $n=10$ & $n=15$ & $n=1$ & $n=5$ & $n=7$ & $n=10$ & $n=15$ \\
\hline & \multicolumn{10}{|c|}{$\rho_{0}=\rho_{1}=-0.8$} \\
\hline 0.90 & 1.0 & 1.0 & 1.0 & 1.0 & 1.0 & 112.1 & 42.1 & 32.0 & 23.2 & 15.4 \\
\hline 0.95 & 1.8 & 1.0 & 1.0 & 1.0 & 1.0 & 150.7 & 90.5 & 77.8 & 64.7 & 50.8 \\
\hline 0.98 & 15.3 & 2.4 & 1.7 & 1.3 & 1.1 & 178.9 & 145.2 & 136.3 & 126.0 & 113.4 \\
\hline 0.99 & 49.1 & 12.2 & 8.3 & 5.4 & 3.3 & 189.2 & 170.3 & 164.9 & 158.5 & 150.1 \\
\hline 1.01 & 49.8 & 12.5 & 8.5 & 5.6 & 3.4 & 189.3 & 170.6 & 165.3 & 158.8 & 150.6 \\
\hline 1.02 & 16.0 & 2.5 & 1.7 & 1.3 & 1.1 & 179.3 & 146.1 & 137.3 & 127.1 & 114.6 \\
\hline 1.05 & 2.0 & 1.0 & 1.0 & 1.0 & 1.0 & 152.8 & 93.9 & 81.3 & 68.2 & 54.0 \\
\hline \multirow[t]{2}{*}{1.10} & 1.0 & 1.0 & 1.0 & 1.0 & 1.0 & 118.4 & 48.3 & 37.5 & 27.7 & 18.8 \\
\hline & \multicolumn{10}{|c|}{$\bar{\rho}_{0}=\rho_{1}=-0.4$} \\
\hline 0.90 & 1.0 & 1.0 & 1.0 & 1.0 & 1.0 & 105.5 & 35.2 & 26.0 & 18.3 & 11.8 \\
\hline 0.95 & 1.5 & 1.0 & 1.0 & 1.0 & 1.0 & 146.4 & 82.1 & 69.3 & 56.4 & 43.1 \\
\hline 0.98 & 11.7 & 1.8 & 1.4 & 1.1 & 1.0 & 176.8 & 139.5 & 129.8 & 118.8 & 105.5 \\
\hline 0.99 & 41.5 & 9.2 & 6.2 & 4.0 & 2.5 & 188.1 & 166.9 & 160.9 & 153.8 & 144.7 \\
\hline 1.01 & 42.1 & 9.4 & 6.3 & 4.1 & 2.5 & 188.2 & 167.2 & 161.3 & 154.2 & 145.1 \\
\hline 1.02 & 12.2 & 1.9 & 1.4 & 1.1 & 1.0 & 177.3 & 140.5 & 130.9 & 120.0 & 106.8 \\
\hline 1.05 & 1.6 & 1.0 & 1.0 & 1.0 & 1.0 & 148.6 & 85.6 & 72.8 & 59.8 & 46.1 \\
\hline \multirow[t]{2}{*}{1.10} & 1.0 & 1.0 & 1.0 & 1.0 & 1.0 & 112.1 & 40.9 & 30.9 & 22.2 & 14.6 \\
\hline & \multicolumn{10}{|c|}{$\rho_{0}=\rho_{1}=0.0$} \\
\hline 0.90 & 1.0 & 1.0 & 1.0 & 1.0 & 1.0 & 95.7 & 26.8 & 19.1 & 12.9 & 8.0 \\
\hline 0.95 & 1.2 & 1.0 & 1.0 & 1.0 & 1.0 & 139.7 & 70.7 & 58.1 & 45.8 & 33.7 \\
\hline 0.98 & 8.0 & 1.4 & 1.1 & 1.0 & 1.0 & 173.6 & 131.0 & 120.4 & 108.5 & 94.4 \\
\hline 0.99 & 32.2 & 6.2 & 4.1 & 2.7 & 1.8 & 186.4 & 161.7 & 154.9 & 146.8 & 136.6 \\
\hline 1.01 & 32.7 & 6.3 & 4.2 & 2.8 & 1.8 & 186.5 & 162.0 & 155.2 & 147.2 & 137.1 \\
\hline 1.02 & 8.4 & 1.4 & 1.1 & 1.0 & 1.0 & 174.1 & 132.1 & 121.6 & 109.8 & 95.8 \\
\hline 1.05 & 1.2 & 1.0 & 1.0 & 1.0 & 1.0 & 142.1 & 74.2 & 61.4 & 48.9 & 36.4 \\
\hline \multirow[t]{2}{*}{1.10} & 1.0 & 1.0 & 1.0 & 1.0 & 1.0 & 102.7 & 31.8 & 23.1 & 16.0 & 10.1 \\
\hline & \multicolumn{10}{|c|}{$\rho_{0}=\rho_{1}=0.4$} \\
\hline 0.90 & 1.0 & 1.0 & 1.0 & 1.0 & 1.0 & 79.3 & 16.5 & 11.1 & 7.2 & 4.3 \\
\hline 0.95 & 1.0 & 1.0 & 1.0 & 1.0 & 1.0 & 127.5 & 53.7 & 42.0 & 31.5 & 21.7 \\
\hline 0.98 & 4.3 & 1.1 & 1.0 & 1.0 & 1.0 & 167.6 & 116.5 & 104.6 & 91.7 & 76.9 \\
\hline 0.99 & 20.6 & 3.3 & 2.3 & 1.6 & 1.2 & 183.2 & 152.3 & 144.1 & 134.5 & 122.7 \\
\hline 1.01 & 21.0 & 3.4 & 2.3 & 1.6 & 1.2 & 183.3 & 152.7 & 144.6 & 135.1 & 123.3 \\
\hline 1.02 & 4.5 & 1.1 & 1.0 & 1.0 & 1.0 & 168.1 & 117.7 & 105.9 & 93.0 & 78.3 \\
\hline 1.05 & 1.0 & 1.0 & 1.0 & 1.0 & 1.0 & 130.4 & 57.0 & 45.1 & 34.1 & 23.8 \\
\hline \multirow[t]{2}{*}{1.10} & 1.0 & 1.0 & 1.0 & 1.0 & 1.0 & 86.6 & 20.2 & 13.9 & 9.1 & 5.5 \\
\hline & \multicolumn{10}{|c|}{$\rho_{0}=\rho_{1}=0.8$} \\
\hline 0.90 & 1.0 & 1.0 & 1.0 & 1.0 & 1.0 & 42.3 & 4.6 & 3.0 & 2.0 & 1.4 \\
\hline 0.95 & 1.0 & 1.0 & 1.0 & 1.0 & 1.0 & 93.8 & 23.3 & 16.2 & 10.8 & 6.6 \\
\hline 0.98 & 1.4 & 1.0 & 1.0 & 1.0 & 1.0 & 148.6 & 80.2 & 67.1 & 54.2 & 40.9 \\
\hline 0.99 & 6.2 & 1.2 & 1.1 & 1.0 & 1.0 & 172.6 & 125.7 & 114.3 & 101.9 & 87.3 \\
\hline 1.01 & 6.3 & 1.2 & 1.1 & 1.0 & 1.0 & 172.8 & 126.2 & 115.0 & 102.5 & 88.0 \\
\hline 1.02 & 1.4 & 1.0 & 1.0 & 1.0 & 1.0 & 149.5 & 81.6 & 68.5 & 55.5 & 42.1 \\
\hline 1.05 & 1.0 & 1.0 & 1.0 & 1.0 & 1.0 & 97.3 & 25.5 & 18.0 & 12.0 & 7.4 \\
\hline 1.10 & 1.0 & 1.0 & 1.0 & 1.0 & 1.0 & 49.0 & 5.9 & 3.8 & 2.4 & 1.6 \\
\hline
\end{tabular}


Table 3: $A R L$ of the one-sided Shewhart-RZ control charts for $\gamma_{X} \in\{0.01,0.2\}, \gamma_{Y} \in$ $\{0.01,0.2\}, \gamma_{X} \neq \gamma_{Y}, \rho_{0} \in\{-0.8,-0.4,0,0.4,0.8\}, \rho_{0}=\rho_{1}, n \in\{1,5,7,10,15\}$.

\begin{tabular}{|c|c|c|c|c|c|c|c|c|c|c|}
\hline \multirow[b]{2}{*}{$\tau$} & \multicolumn{5}{|c|}{$\left(\gamma_{X}=0.01, \gamma_{Y}=0.2\right)$} & \multicolumn{5}{|c|}{$\left(\gamma_{X}=0.2, \gamma_{Y}=0.01\right)$} \\
\hline & $n=1$ & $n=5$ & $n=7$ & $n=10$ & $n=15$ & $n=1$ & $n=5$ & $n=7$ & $n=10$ & $n=15$ \\
\hline & \multicolumn{10}{|c|}{$\rho_{0}=\rho_{1}=-0.8$} \\
\hline 0.90 & 31.7 & 9.6 & 6.9 & 4.8 & 3.1 & 96.3 & 20.3 & 13.2 & 8.1 & 4.6 \\
\hline 0.95 & 75.4 & 36.4 & 29.1 & 22.3 & 15.7 & 140.3 & 61.4 & 47.6 & 35.1 & 23.6 \\
\hline 0.98 & 133.6 & 96.7 & 87.1 & 76.6 & 64.3 & 174.0 & 123.9 & 111.0 & 96.9 & 80.7 \\
\hline 0.99 & 163.1 & 138.0 & 130.7 & 122.1 & 111.2 & 186.6 & 157.2 & 148.7 & 138.7 & 126.1 \\
\hline 1.01 & 186.8 & 157.6 & 149.1 & 139.2 & 126.6 & 163.4 & 138.5 & 131.2 & 122.6 & 111.9 \\
\hline 1.02 & 174.5 & 125.0 & 112.3 & 98.3 & 82.1 & 134.7 & 98.0 & 88.5 & 77.9 & 65.7 \\
\hline 1.05 & 142.7 & 64.8 & 50.8 & 37.9 & 25.8 & 78.8 & 39.2 & 31.6 & 24.3 & 17.4 \\
\hline \multirow[t]{2}{*}{1.10} & 103.3 & 24.6 & 16.4 & 10.3 & 5.9 & 36.8 & 11.9 & 8.7 & 6.1 & 3.9 \\
\hline & \multicolumn{10}{|c|}{$\rho_{0}=\rho_{1}=-0.4$} \\
\hline 0.90 & 30.4 & 9.2 & 6.6 & 4.6 & 3.0 & 95.6 & 19.6 & 12.7 & 7.8 & 4.4 \\
\hline 0.95 & 73.6 & 35.2 & 28.1 & 21.4 & 15.1 & 139.8 & 60.3 & 46.6 & 34.2 & 22.9 \\
\hline 0.98 & 132.2 & 95.2 & 85.6 & 75.1 & 62.9 & 173.8 & 123.0 & 110.0 & 95.8 & 79.6 \\
\hline 0.99 & 162.2 & 136.9 & 129.5 & 120.8 & 109.9 & 186.5 & 156.7 & 148.0 & 137.9 & 125.1 \\
\hline 1.01 & 186.6 & 157.1 & 148.5 & 138.4 & 125.7 & 162.5 & 137.4 & 130.0 & 121.4 & 110.5 \\
\hline 1.02 & 174.3 & 124.2 & 111.3 & 97.2 & 81.0 & 133.2 & 96.5 & 87.0 & 76.5 & 64.3 \\
\hline 1.05 & 142.3 & 63.8 & 49.8 & 37.0 & 25.1 & 76.9 & 37.9 & 30.5 & 23.4 & 16.7 \\
\hline \multirow[t]{2}{*}{1.10} & 102.6 & 23.9 & 15.9 & 9.9 & 5.7 & 35.4 & 11.4 & 8.3 & 5.8 & 3.8 \\
\hline & \multicolumn{10}{|c|}{$\rho_{0}=\rho_{1}=0.0$} \\
\hline 0.90 & 29.1 & 8.7 & 6.3 & 4.4 & 2.9 & 94.9 & 19.0 & 12.2 & 7.4 & 4.2 \\
\hline 0.95 & 71.6 & 34.0 & 27.1 & 20.6 & 14.4 & 139.3 & 59.3 & 45.6 & 33.3 & 22.1 \\
\hline 0.98 & 130.6 & 93.6 & 84.0 & 73.6 & 61.5 & 173.5 & 122.1 & 109.0 & 94.7 & 78.4 \\
\hline 0.99 & 161.2 & 135.6 & 128.2 & 119.5 & 108.5 & 186.4 & 156.1 & 147.3 & 137.0 & 124.1 \\
\hline 1.01 & 186.5 & 156.5 & 147.8 & 137.5 & 124.7 & 161.5 & 136.1 & 128.7 & 120.0 & 109.1 \\
\hline 1.02 & 174.0 & 123.3 & 110.3 & 96.1 & 79.8 & 131.7 & 94.9 & 85.4 & 74.9 & 62.8 \\
\hline 1.05 & 141.8 & 62.7 & 48.8 & 36.0 & 24.3 & 75.0 & 36.6 & 29.4 & 22.5 & 16.0 \\
\hline \multirow[t]{2}{*}{1.10} & 101.9 & 23.2 & 15.3 & 9.5 & 5.4 & 34.0 & 10.9 & 7.9 & 5.5 & 3.6 \\
\hline & \multicolumn{10}{|c|}{$\rho_{0}=\rho_{1}=0.4$} \\
\hline 0.90 & 27.8 & 8.3 & 6.0 & 4.1 & 2.7 & 94.1 & 18.4 & 11.7 & 7.1 & 4.0 \\
\hline 0.95 & 69.6 & 32.7 & 26.0 & 19.7 & 13.8 & 138.8 & 58.2 & 44.6 & 32.3 & 21.4 \\
\hline 0.98 & 128.9 & 91.9 & 82.4 & 72.0 & 60.0 & 173.3 & 121.2 & 107.9 & 93.5 & 77.1 \\
\hline 0.99 & 160.1 & 134.3 & 126.8 & 118.0 & 107.0 & 186.2 & 155.5 & 146.6 & 136.1 & 123.1 \\
\hline 1.01 & 186.4 & 155.9 & 147.0 & 136.7 & 123.7 & 160.5 & 134.9 & 127.4 & 118.6 & 107.7 \\
\hline 1.02 & 173.8 & 122.3 & 109.2 & 94.9 & 78.5 & 130.0 & 93.2 & 83.8 & 73.3 & 61.3 \\
\hline 1.05 & 141.3 & 61.6 & 47.7 & 35.0 & 23.5 & 73.0 & 35.3 & 28.3 & 21.6 & 15.2 \\
\hline \multirow[t]{2}{*}{1.10} & 101.2 & 22.5 & 14.7 & 9.1 & 5.2 & 32.5 & 10.3 & 7.5 & 5.2 & 3.4 \\
\hline & \multicolumn{10}{|c|}{$\rho_{0}=\rho_{1}=0.8$} \\
\hline 0.90 & 26.4 & 7.8 & 5.6 & 3.9 & 2.6 & 93.4 & 17.7 & 11.3 & 6.8 & 3.8 \\
\hline 0.95 & 67.5 & 31.5 & 24.9 & 18.8 & 13.1 & 138.3 & 57.1 & 43.5 & 31.4 & 20.6 \\
\hline 0.98 & 127.2 & 90.1 & 80.7 & 70.3 & 58.4 & 173.0 & 120.2 & 106.8 & 92.3 & 75.8 \\
\hline 0.99 & 159.0 & 133.0 & 125.4 & 116.6 & 105.5 & 186.1 & 154.9 & 145.8 & 135.2 & 122.0 \\
\hline 1.01 & 186.2 & 155.3 & 146.3 & 135.7 & 122.6 & 159.3 & 133.5 & 126.0 & 117.2 & 106.1 \\
\hline 1.02 & 173.5 & 121.4 & 108.1 & 93.7 & 77.2 & 128.3 & 91.5 & 82.0 & 71.6 & 59.7 \\
\hline 1.05 & 140.8 & 60.5 & 46.6 & 34.0 & 22.7 & 70.8 & 34.0 & 27.1 & 20.7 & 14.5 \\
\hline 1.10 & 100.4 & 21.7 & 14.2 & 8.7 & 4.9 & 31.0 & 9.8 & 7.1 & 4.9 & 3.2 \\
\hline
\end{tabular}


Table 4: $A R L$ of the one-sided Shewhart-RZ control charts for $\gamma_{X} \in\{0.01,0.2\}, \gamma_{Y} \in$ $\{0.01,0.2\}, \gamma_{X}=\gamma_{Y}, \rho_{0} \in\{-0.8,-0.4,0,0.4,0.8\}, \rho_{0} \neq \rho_{1}, n \in\{1,5,7,10,15\}$.

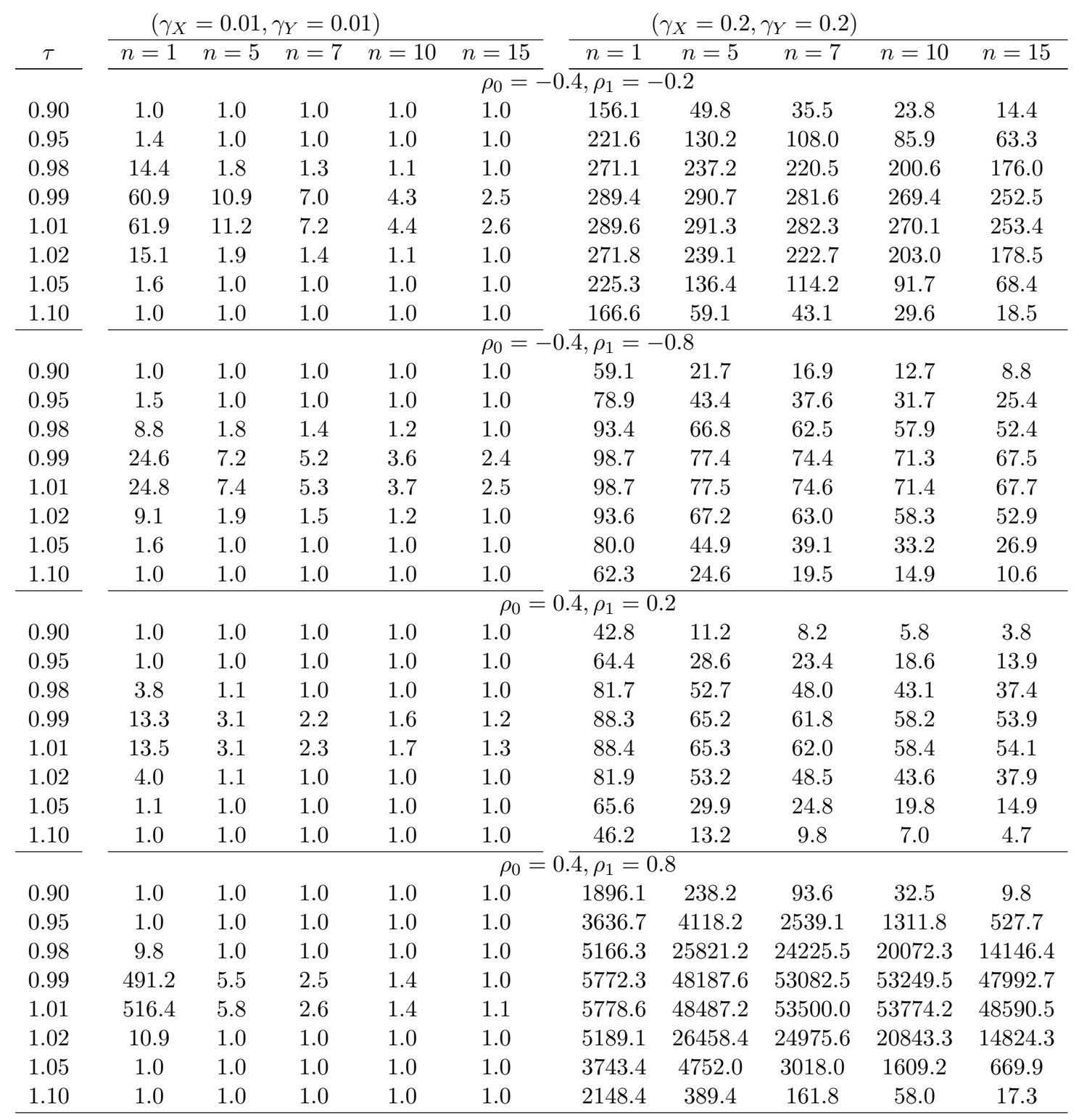


Table 5: $A R L$ of the one-sided Shewhart-RZ control charts for $\gamma_{X} \in\{0.01,0.2\}, \gamma_{Y} \in$ $\{0.01,0.2\}, \gamma_{X} \neq \gamma_{Y}, \rho_{0} \in\{-0.8,-0.4,0,0.4,0.8\}, \rho_{0} \neq \rho_{1}, n \in\{1,5,7,10,15\}$.

\begin{tabular}{|c|c|c|c|c|c|c|c|c|c|c|}
\hline \multirow[b]{2}{*}{$\tau$} & \multicolumn{5}{|c|}{$\left(\gamma_{X}=0.01, \gamma_{Y}=0.2\right)$} & \multicolumn{5}{|c|}{$\left(\gamma_{X}=0.2, \gamma_{Y}=0.01\right)$} \\
\hline & $n=1$ & $n=5$ & $n=7$ & $n=10$ & $n=15$ & $n=1$ & $n=5$ & $n=7$ & $n=10$ & $n=15$ \\
\hline & \multicolumn{10}{|c|}{$\rho_{0}=-0.4, \overline{\rho_{1}}=-0.2$} \\
\hline 0.90 & 32.2 & 9.4 & 6.7 & 4.6 & 3.0 & 98.7 & 20.2 & 13.0 & 7.9 & 4.4 \\
\hline 0.95 & 79.6 & 37.0 & 29.4 & 22.2 & 15.5 & 144.6 & 62.9 & 48.5 & 35.5 & 23.6 \\
\hline 0.98 & 145.6 & 102.3 & 91.6 & 80.0 & 66.7 & 179.9 & 129.4 & 115.8 & 100.9 & 83.7 \\
\hline 0.99 & 179.9 & 148.3 & 139.8 & 130.0 & 117.8 & 193.2 & 165.3 & 156.4 & 145.8 & 132.4 \\
\hline 1.01 & 193.3 & 165.7 & 156.8 & 146.3 & 133.0 & 180.2 & 148.9 & 140.4 & 130.6 & 118.5 \\
\hline 1.02 & 180.4 & 130.6 & 117.2 & 102.3 & 85.2 & 146.8 & 103.7 & 93.1 & 81.5 & 68.2 \\
\hline 1.05 & 147.2 & 66.6 & 51.9 & 38.4 & 25.9 & 83.4 & 39.9 & 31.9 & 24.4 & 17.2 \\
\hline \multirow[t]{2}{*}{1.10} & 106.0 & 24.7 & 16.3 & 10.1 & 5.7 & 37.6 & 11.7 & 8.5 & 5.9 & 3.8 \\
\hline & \multicolumn{10}{|c|}{$\rho_{0}=-0.4, \overline{\rho_{1}=-0.8}$} \\
\hline 0.90 & 27.5 & 8.8 & 6.4 & 4.5 & 3.0 & 89.8 & 18.6 & 12.2 & 7.5 & 4.3 \\
\hline 0.95 & 63.5 & 32.1 & 25.9 & 19.9 & 14.2 & 130.9 & 55.7 & 43.2 & 31.8 & 21.5 \\
\hline 0.98 & 110.5 & 83.2 & 75.4 & 66.7 & 56.4 & 162.4 & 111.7 & 99.8 & 86.9 & 72.3 \\
\hline 0.99 & 134.0 & 117.7 & 112.1 & 105.2 & 96.4 & 174.2 & 141.5 & 133.3 & 123.9 & 112.3 \\
\hline 1.01 & 174.3 & 141.8 & 133.6 & 124.3 & 112.8 & 134.3 & 118.1 & 112.5 & 105.7 & 96.9 \\
\hline 1.02 & 162.8 & 112.7 & 100.9 & 88.1 & 73.6 & 111.3 & 84.3 & 76.5 & 67.8 & 57.5 \\
\hline 1.05 & 133.2 & 58.8 & 46.1 & 34.4 & 23.5 & 66.3 & 34.4 & 28.0 & 21.7 & 15.7 \\
\hline \multirow[t]{2}{*}{1.10} & 96.3 & 22.6 & 15.1 & 9.5 & 5.5 & 31.8 & 10.8 & 8.0 & 5.6 & 3.7 \\
\hline & \multicolumn{10}{|c|}{$\rho_{0}=0.4, \overline{\rho_{1}}=0.2$} \\
\hline 0.90 & 26.3 & 8.1 & 5.9 & 4.1 & 2.7 & 91.0 & 17.9 & 11.5 & 7.0 & 4.0 \\
\hline 0.95 & 64.2 & 31.1 & 24.9 & 19.0 & 13.4 & 134.0 & 55.7 & 42.8 & 31.1 & 20.7 \\
\hline 0.98 & 116.6 & 85.3 & 76.8 & 67.4 & 56.5 & 167.1 & 115.0 & 102.4 & 88.7 & 73.2 \\
\hline 0.99 & 143.8 & 123.6 & 117.1 & 109.4 & 99.6 & 179.6 & 147.1 & 138.4 & 128.4 & 116.1 \\
\hline 1.01 & 179.7 & 147.5 & 138.9 & 128.9 & 116.6 & 144.1 & 124.1 & 117.6 & 109.9 & 100.2 \\
\hline 1.02 & 167.6 & 116.1 & 103.6 & 90.0 & 74.6 & 117.6 & 86.5 & 78.1 & 68.7 & 57.7 \\
\hline 1.05 & 136.4 & 59.0 & 45.7 & 33.7 & 22.7 & 67.2 & 33.5 & 27.0 & 20.8 & 14.8 \\
\hline \multirow[t]{2}{*}{1.10} & 97.8 & 21.8 & 14.4 & 8.9 & 5.1 & 30.6 & 10.0 & 7.3 & 5.1 & 3.4 \\
\hline & \multicolumn{10}{|c|}{$\rho_{0}=0.4, \overline{\rho_{1}}=0.8$} \\
\hline 0.90 & 31.3 & 8.7 & 6.2 & 4.2 & 2.7 & 100.9 & 19.5 & 12.3 & 7.4 & 4.1 \\
\hline 0.95 & 83.1 & 36.5 & 28.6 & 21.3 & 14.7 & 149.3 & 63.7 & 48.6 & 35.0 & 22.9 \\
\hline 0.98 & 160.7 & 107.8 & 95.7 & 82.7 & 68.1 & 186.7 & 135.2 & 120.7 & 104.6 & 86.0 \\
\hline 0.99 & 202.7 & 160.8 & 150.6 & 139.0 & 124.9 & 200.8 & 174.6 & 165.3 & 153.9 & 139.3 \\
\hline 1.01 & 201.0 & 175.1 & 165.8 & 154.5 & 140.0 & 203.1 & 161.4 & 151.2 & 139.7 & 125.7 \\
\hline 1.02 & 187.3 & 136.6 & 122.2 & 106.1 & 87.6 & 162.1 & 109.5 & 97.4 & 84.4 & 69.7 \\
\hline 1.05 & 152.0 & 67.6 & 52.1 & 38.0 & 25.2 & 87.4 & 39.5 & 31.2 & 23.5 & 16.3 \\
\hline 1.10 & 108.5 & 24.0 & 15.6 & 9.5 & 5.3 & 37.0 & 10.9 & 7.8 & 5.4 & 3.5 \\
\hline
\end{tabular}


Figure 2: The effect of $\eta_{X}$ and $\eta_{Y}$ on the overall performance of the Shewhart-RZ control chart in the presence of measurement error for $\theta_{X}=$ $\theta_{Y}=0, \rho_{M}=0, n \in\{1,15\}, \gamma_{X}=\gamma_{Y} \in\{0.01,0.2\}$, and $\rho_{0}=\rho_{1}$.

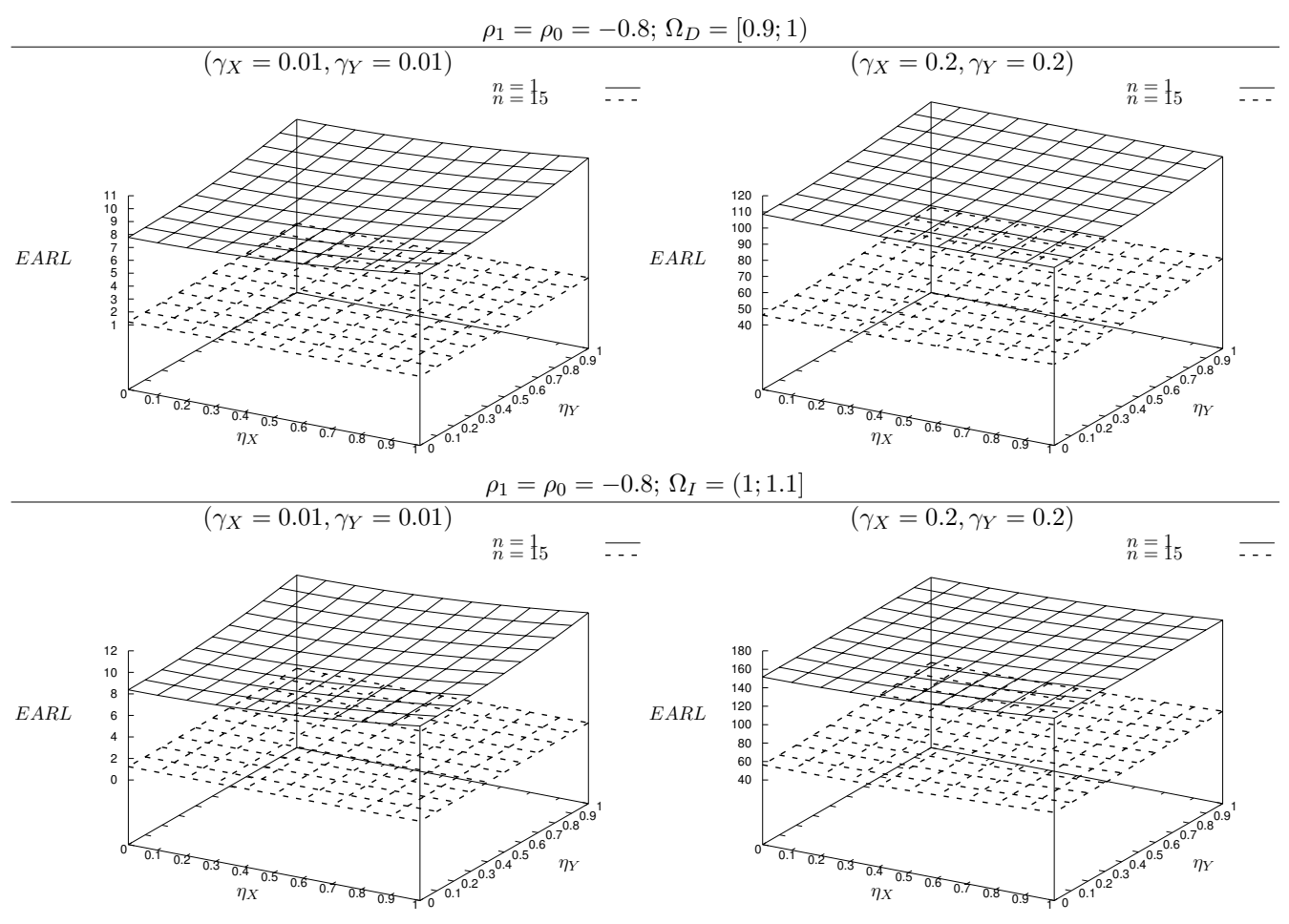


Figure 3: The effect of $\eta_{X}$ and $\eta_{Y}$ on the overall performance of the Shewhart-RZ control chart in the presence of measurement error for $\theta_{X}=$ $\theta_{Y}=0, \rho_{M}=0, n \in\{1,15\}, \gamma_{X}=\gamma_{Y} \in\{0.01,0.2\}$, and $\rho_{0} \neq \rho_{1}$.
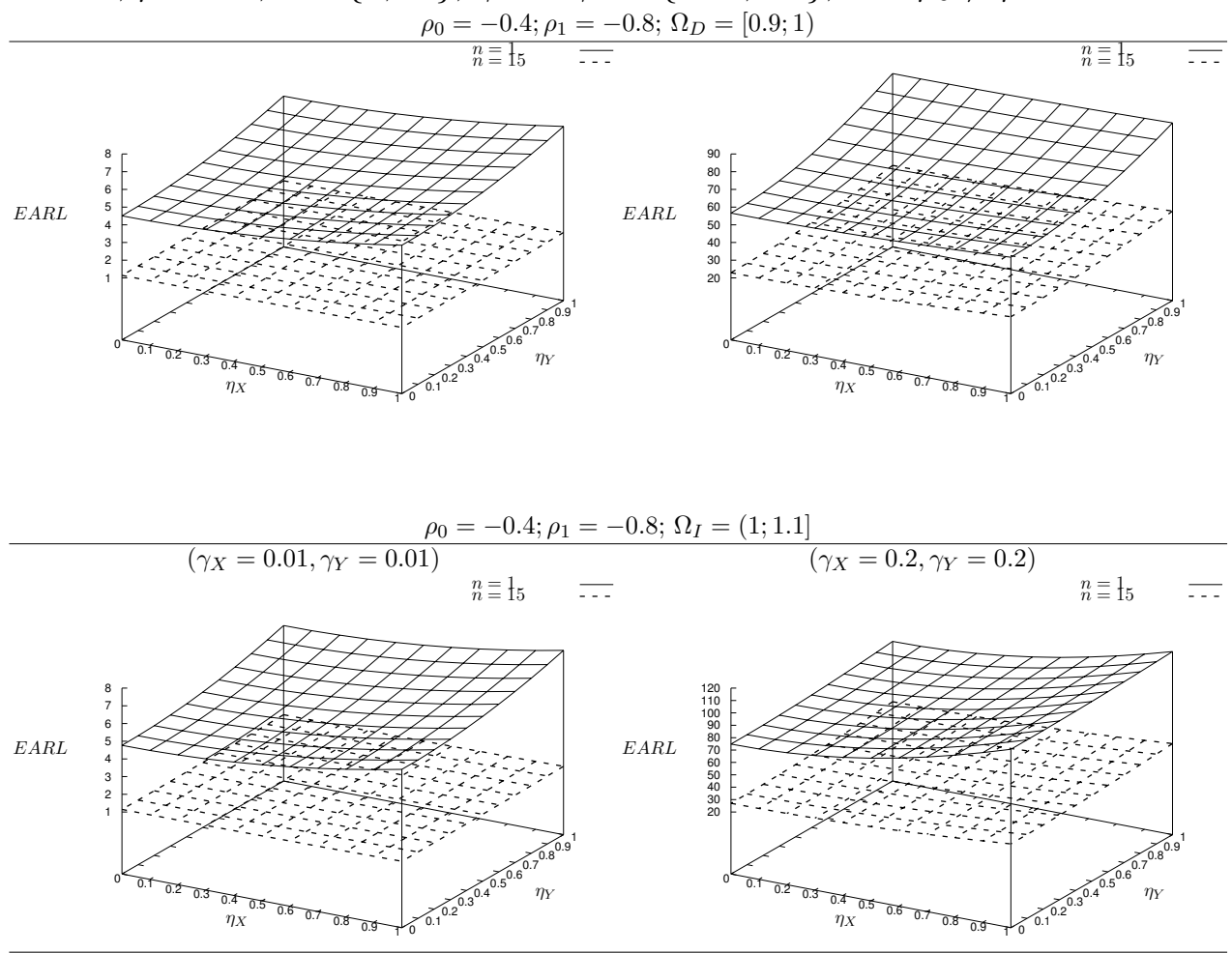
Figure 4: The effect of $\theta_{X}$ and $\theta_{Y}$ on the overall performance of the Shewhart-RZ control chart in the presence of measurement error for $\eta_{X}=$ $\eta_{Y}=0, \rho_{M}=0, n \in\{1,15\}, \gamma_{X}=\gamma_{Y} \in\{0.01,0.2\}$, and $\rho_{0}=\rho_{1}$.
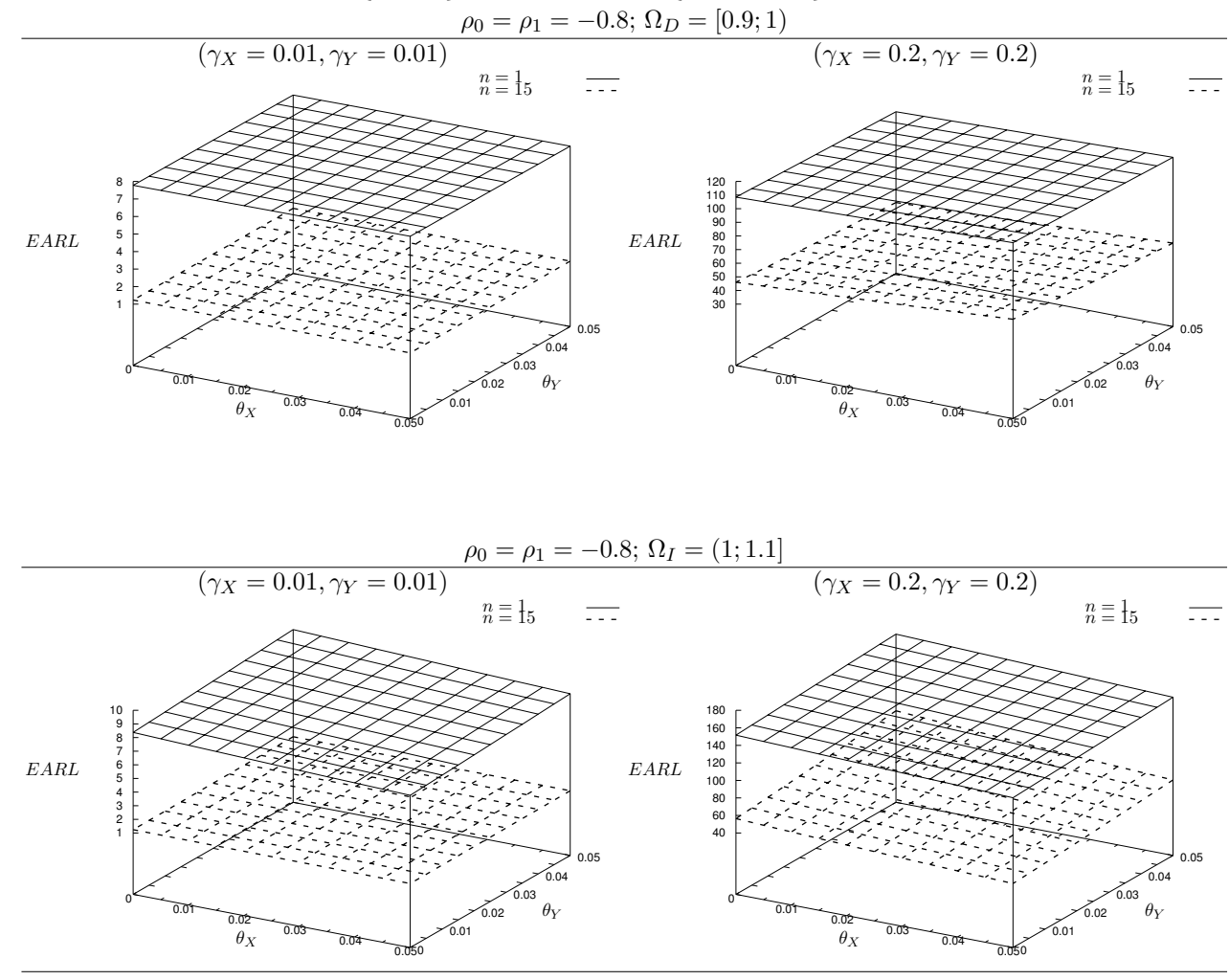
Figure 5: The effect of $\theta_{X}$ and $\theta_{Y}$ on the overall performance of the Shewhart-RZ control chart in the presence of measurement error for $\eta_{X}=$ $\eta_{Y}=0, \rho_{M}=0, n \in\{1,15\}, \gamma_{X}=\gamma_{Y} \in\{0.01,0.2\}$, and $\rho_{0} \neq \rho_{1}$.

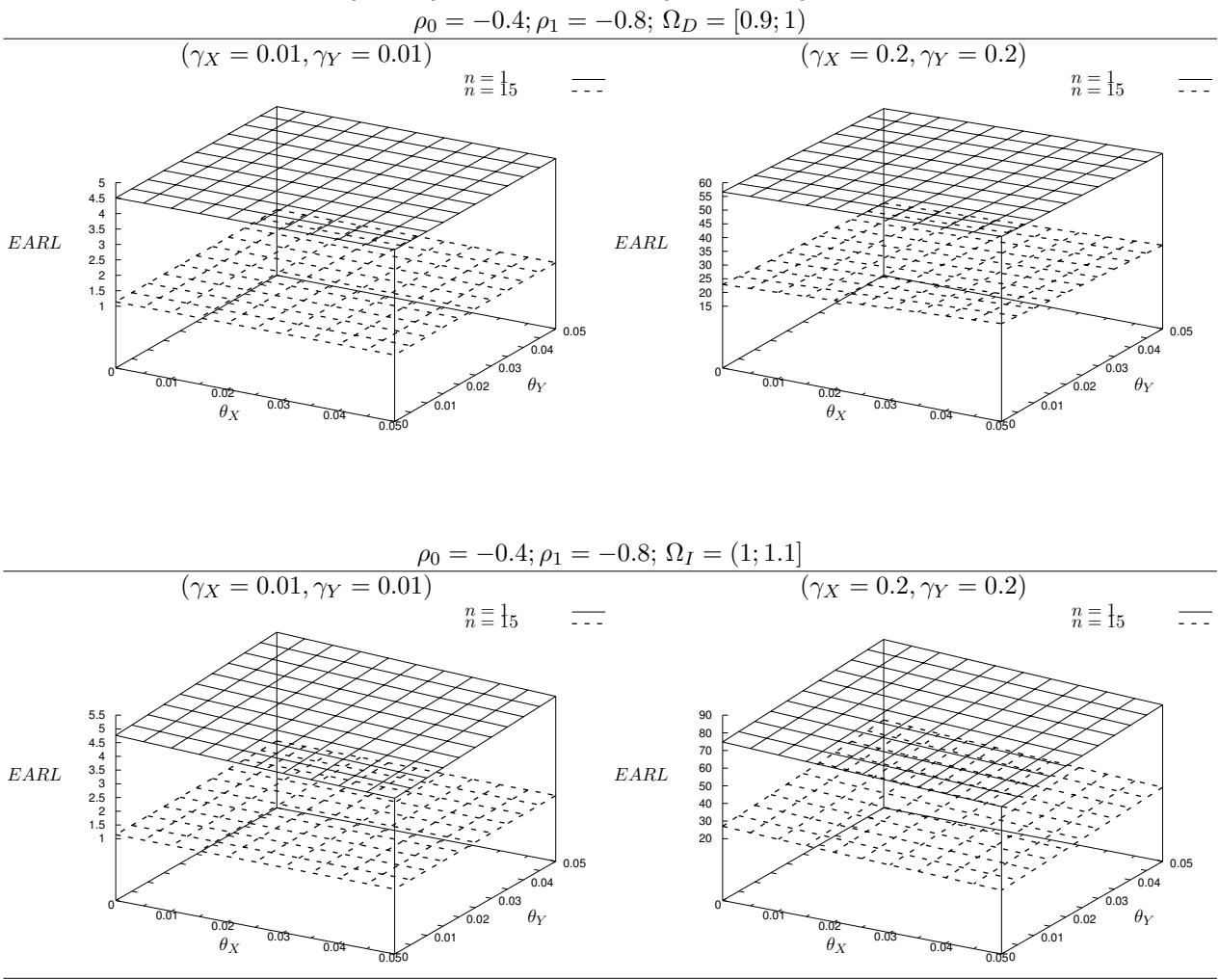


Figure 6: The effect of $\rho_{M}$ on the overall performance of the Shewhart-RZ control chart in the presence of measurement error for $n=1$ (-口-) and $n=15$ $(-\mathbf{m}), \eta_{X}=\eta_{Y}=0.28, \theta_{X}=\theta_{Y}=0.05, n \in\{1,15\}, \gamma_{X}=\gamma_{Y} \in\{0.01,0.2\}$ and $\rho_{0}=\rho_{1}=-0.8$.
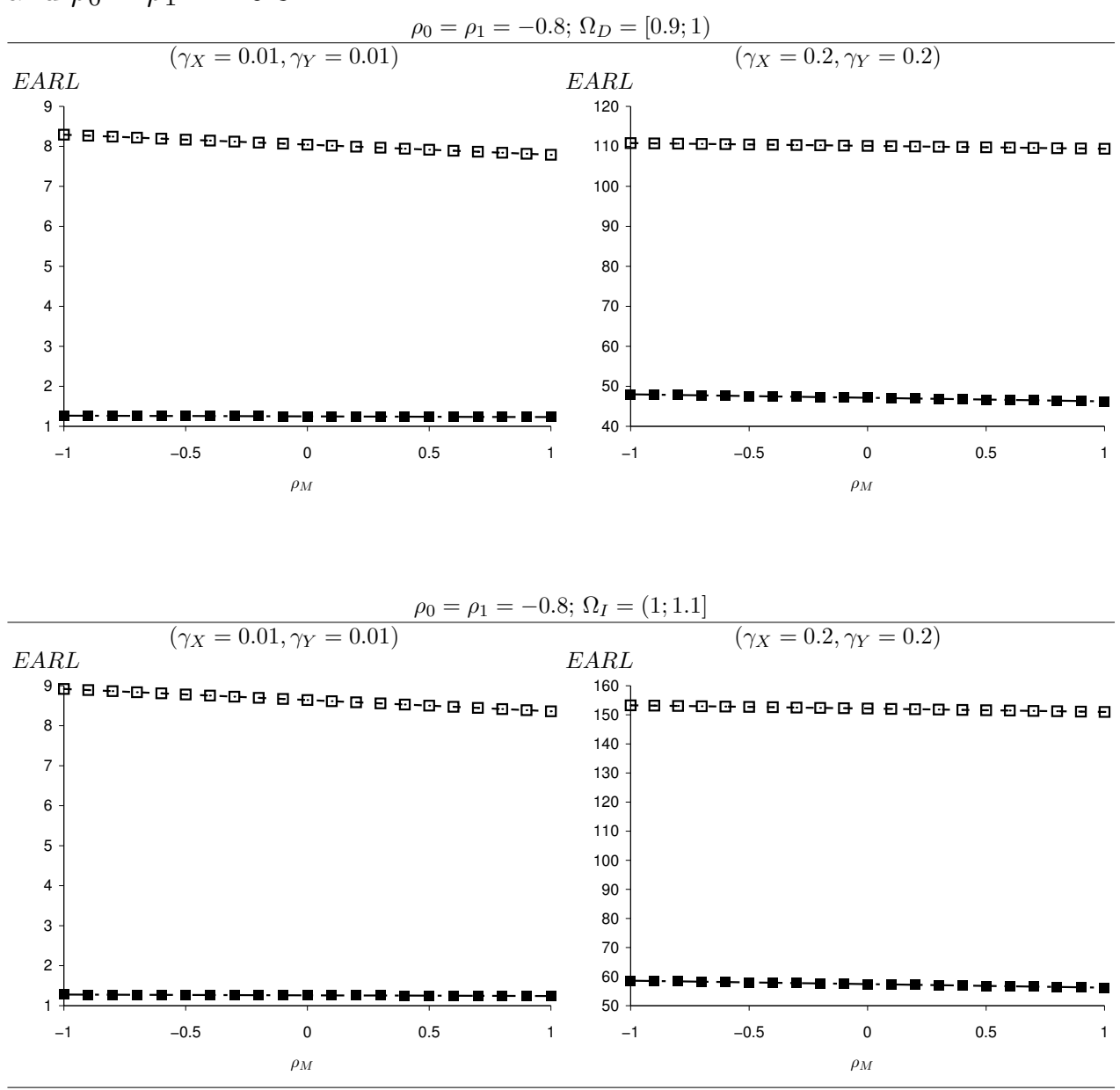
Figure 7: The effect of $\rho_{M}$ on the overall performance of the Shewhart-RZ control chart in the presence of measurement error for $n=1$ (-口-) and $n=15$ $(-\mathbf{-}), \eta_{X}=\eta_{Y}=0.28, \theta_{X}=\theta_{Y}=0.05, n \in\{1,15\}, \gamma_{X}=\gamma_{Y} \in\{0.01,0.2\}$ and $\rho_{0}=-0.4, \rho_{1}=-0.8$.
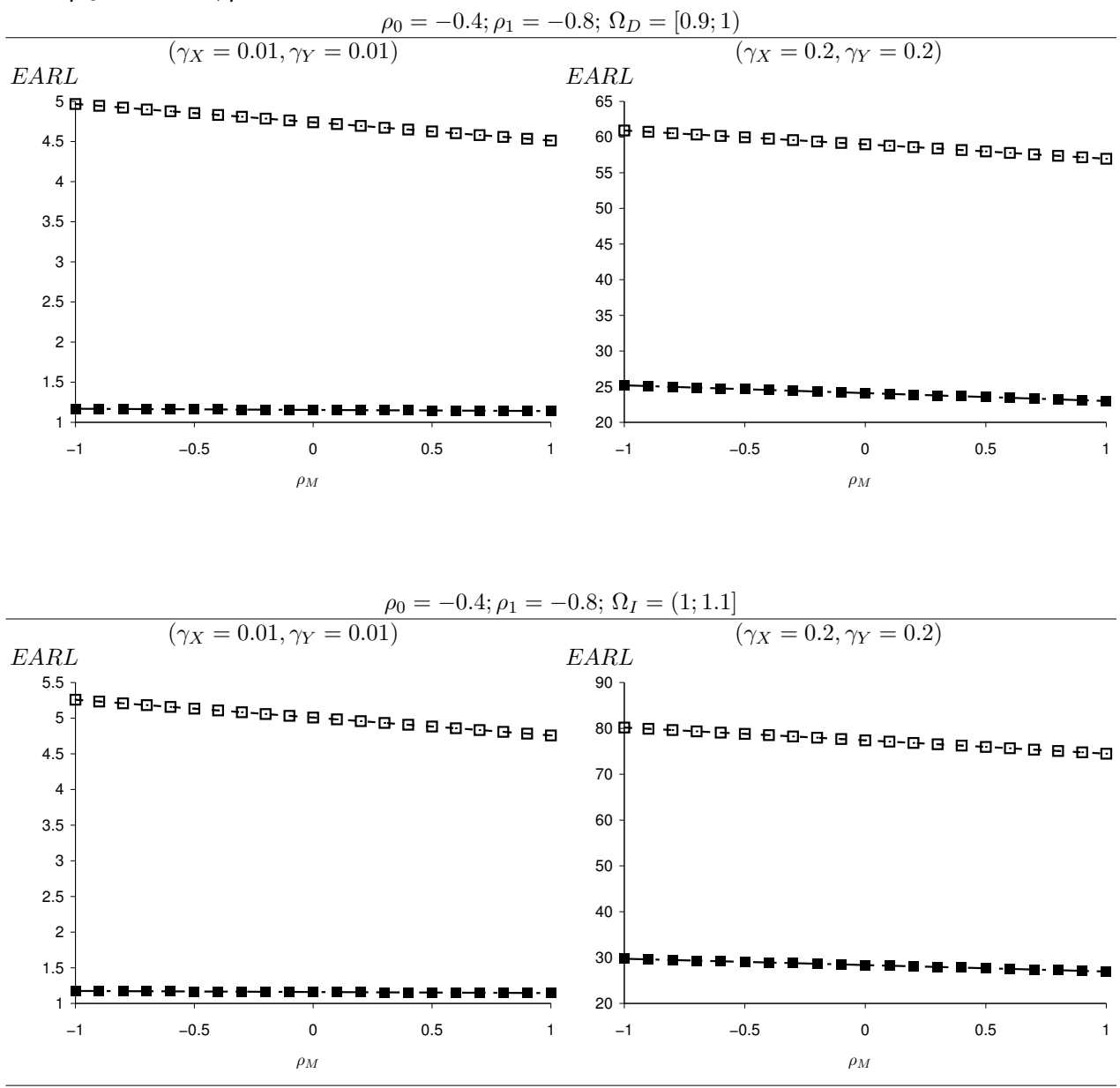
Figure 8: The effect of $m$ on the overall performance of the Shewhart-RZ control chart in the presence of measurement error for $n=1$ (-ם-) and $n=15$ $(-\mathbf{-}), \eta_{X}=\eta_{Y}=0.28, \theta_{X}=\theta_{Y}=0.05, n \in\{1,15\}, \gamma_{X}=\gamma_{Y} \in\{0.01,0.2\}$ and $\rho_{0}=\rho_{1}=-0.8$.
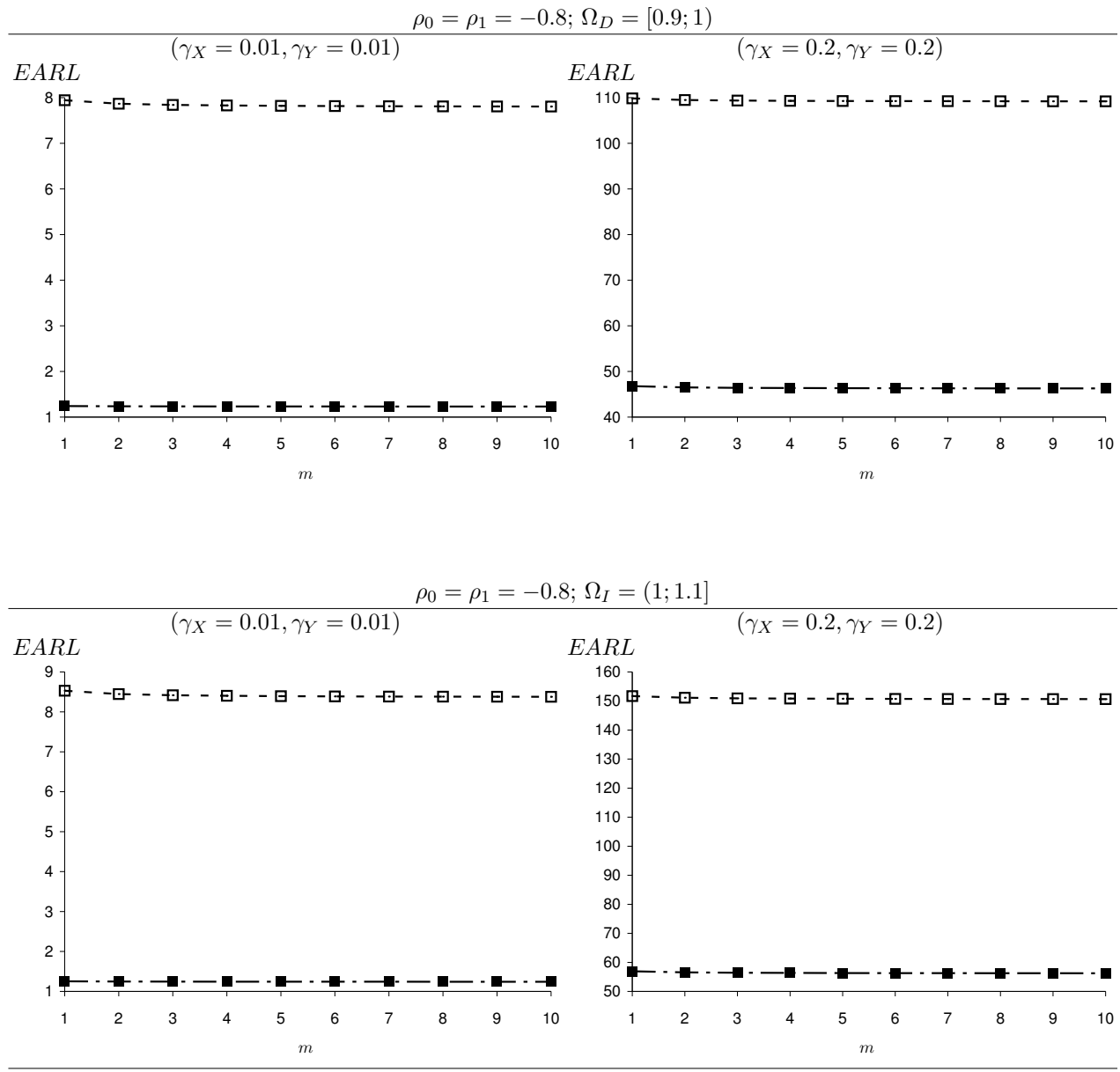
Figure 9: The effect of $m$ on the overall performance of the Shewhart-RZ control chart in the presence of measurement error for $n=1(-\square-)$ and $n=15$ $(-\mathbf{-}), \eta_{X}=\eta_{Y}=0.28, \theta_{X}=\theta_{Y}=0.05, n \in\{1,15\}, \gamma_{X}=\gamma_{Y} \in\{0.01,0.2\}$ and $\rho_{0}=-0.4, \rho_{1}=-0.8$.
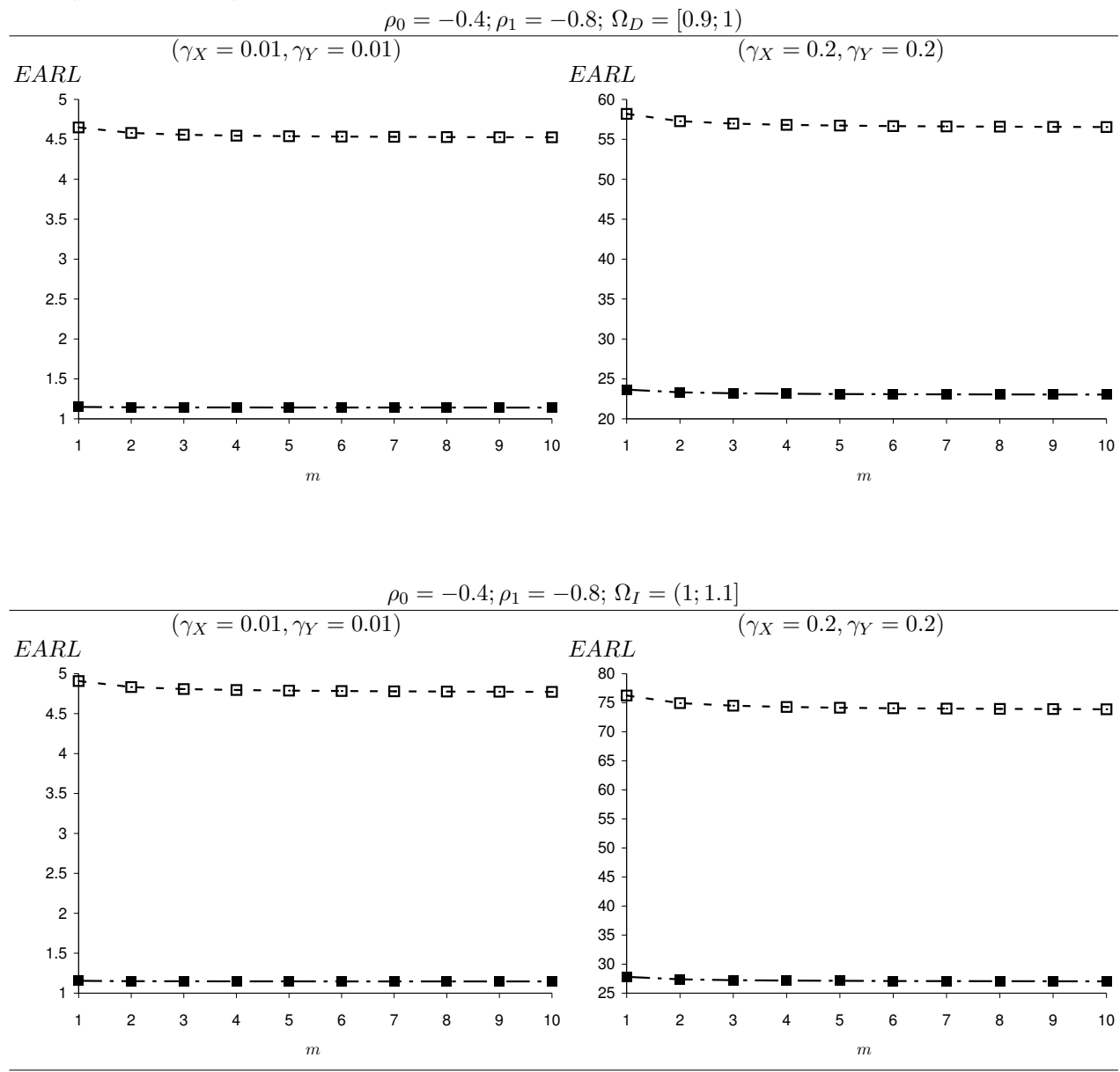
Table 6: The battery recycling industry example data

\begin{tabular}{|c|c|c|c|c|c|c|c|}
\hline Sample & & & $\begin{array}{c}X_{i, j}^{*}[\mathrm{~kg}] \\
Y_{i, j}^{*}[\mathrm{~kg}]\end{array}$ & & & $\begin{array}{c}\bar{X}_{i}^{*}[\mathrm{~kg}] \\
\bar{Y}_{i}^{*}[\mathrm{~kg}]\end{array}$ & $\hat{Z}_{i}^{*}=\frac{\bar{X}_{i}^{*}}{Y_{i}^{*}}$ \\
\hline 1 & $\begin{array}{c}95.864 \\
100.891\end{array}$ & $\begin{array}{c}94.731 \\
100.143\end{array}$ & $\begin{array}{c}9, j .643 \\
100.340\end{array}$ & $\begin{array}{l}94.193 \\
97.740\end{array}$ & $\begin{array}{l}94.328 \\
99.295\end{array}$ & $\begin{array}{l}94.752 \\
99.682\end{array}$ & 0.951 \\
\hline 2 & $\begin{array}{l}94.969 \\
98.903\end{array}$ & $\begin{array}{l}94.935 \\
96.883\end{array}$ & $\begin{array}{l}94.270 \\
97.329\end{array}$ & $\begin{array}{c}94.663 \\
101.021\end{array}$ & $\begin{array}{c}93.961 \\
100.278\end{array}$ & $\begin{array}{l}94.560 \\
98.883\end{array}$ & 0.956 \\
\hline 3 & $\begin{array}{c}93.274 \\
101.525\end{array}$ & $\begin{array}{c}95.927 \\
100.461\end{array}$ & $\begin{array}{c}94.961 \\
100.826\end{array}$ & $\begin{array}{l}96.021 \\
98.936\end{array}$ & $\begin{array}{c}95.429 \\
101.398\end{array}$ & $\begin{array}{c}95.122 \\
100.629\end{array}$ & 0.945 \\
\hline 4 & $\begin{array}{c}95.350 \\
101.137\end{array}$ & $\begin{array}{c}96.128 \\
101.127\end{array}$ & $\begin{array}{c}96.610 \\
100.052\end{array}$ & $\begin{array}{l}95.022 \\
98.796\end{array}$ & $\begin{array}{c}95.737 \\
100.115\end{array}$ & $\begin{array}{c}95.769 \\
100.245\end{array}$ & 0.955 \\
\hline 5 & $\begin{array}{l}94.697 \\
98.996\end{array}$ & $\begin{array}{c}96.827 \\
100.356\end{array}$ & $\begin{array}{l}94.392 \\
99.860\end{array}$ & $\begin{array}{c}94.197 \\
100.082\end{array}$ & $\begin{array}{l}96.205 \\
99.377\end{array}$ & $\begin{array}{l}95.264 \\
99.734\end{array}$ & 0.955 \\
\hline 6 & $\begin{array}{l}94.623 \\
99.309\end{array}$ & $\begin{array}{l}93.930 \\
99.727\end{array}$ & $\begin{array}{l}96.532 \\
98.908\end{array}$ & $\begin{array}{l}94.197 \\
98.534\end{array}$ & $\begin{array}{l}93.573 \\
99.745\end{array}$ & $\begin{array}{l}94.571 \\
99.245\end{array}$ & 0.953 \\
\hline 7 & $\begin{array}{l}94.419 \\
99.936\end{array}$ & $\begin{array}{l}95.445 \\
99.836\end{array}$ & $\begin{array}{c}94.328 \\
101.138\end{array}$ & $\begin{array}{l}95.120 \\
99.668\end{array}$ & $\begin{array}{c}95.880 \\
101.850\end{array}$ & $\begin{array}{c}95.038 \\
100.486\end{array}$ & 0.946 \\
\hline 8 & $\begin{array}{l}96.144 \\
98.212\end{array}$ & $\begin{array}{c}94.121 \\
100.667\end{array}$ & $\begin{array}{l}96.163 \\
99.683\end{array}$ & $\begin{array}{l}93.803 \\
99.044\end{array}$ & $\begin{array}{c}94.900 \\
101.184\end{array}$ & $\begin{array}{l}95.026 \\
99.758\end{array}$ & 0.953 \\
\hline 9 & $\begin{array}{l}94.151 \\
99.173\end{array}$ & $\begin{array}{c}94.786 \\
100.873\end{array}$ & $\begin{array}{c}95.127 \\
100.487\end{array}$ & $\begin{array}{c}94.098 \\
100.635\end{array}$ & $\begin{array}{l}94.307 \\
98.675\end{array}$ & $\begin{array}{l}94.494 \\
99.969\end{array}$ & 0.948 \\
\hline 10 & $\begin{array}{l}96.006 \\
99.392\end{array}$ & $\begin{array}{l}96.319 \\
98.783\end{array}$ & $\begin{array}{c}94.026 \\
100.008\end{array}$ & $\begin{array}{c}94.660 \\
101.235\end{array}$ & $\begin{array}{c}93.948 \\
101.325\end{array}$ & $\begin{array}{c}94.992 \\
100.149\end{array}$ & 0.949 \\
\hline 11 & $\begin{array}{c}93.436 \\
100.491\end{array}$ & $\begin{array}{c}94.988 \\
100.976\end{array}$ & $\begin{array}{c}93.583 \\
100.815\end{array}$ & $\begin{array}{c}94.831 \\
100.102\end{array}$ & $\begin{array}{c}92.875 \\
100.685\end{array}$ & $\begin{array}{l}93.9426 \\
100.614\end{array}$ & 0.934 \\
\hline 12 & $\begin{array}{c}95.832 \\
100.007\end{array}$ & $\begin{array}{c}95.250 \\
101.654\end{array}$ & $\begin{array}{c}94.402 \\
100.648\end{array}$ & $\begin{array}{c}95.221 \\
101.531\end{array}$ & $\begin{array}{l}95.698 \\
99.040\end{array}$ & $\begin{array}{c}95.281 \\
100.576\end{array}$ & 0.947 \\
\hline 13 & $\begin{array}{l}95.746 \\
99.164\end{array}$ & $\begin{array}{c}93.764 \\
100.864\end{array}$ & $\begin{array}{c}92.958 \\
100.174\end{array}$ & $\begin{array}{l}94.811 \\
99.642\end{array}$ & $\begin{array}{c}94.250 \\
100.309\end{array}$ & $\begin{array}{c}94.306 \\
100.031\end{array}$ & 0.943 \\
\hline 14 & $\begin{array}{c}95.897 \\
101.025\end{array}$ & $\begin{array}{l}95.408 \\
98.955\end{array}$ & $\begin{array}{c}95.121 \\
100.267\end{array}$ & $\begin{array}{l}94.810 \\
99.332\end{array}$ & $\begin{array}{c}96.402 \\
100.238\end{array}$ & $\begin{array}{l}95.528 \\
99.963\end{array}$ & 0.956 \\
\hline 15 & $\begin{array}{l}95.481 \\
99.933\end{array}$ & $\begin{array}{l}95.595 \\
99.406\end{array}$ & $\begin{array}{c}94.547 \\
102.736\end{array}$ & $\begin{array}{c}95.299 \\
100.533\end{array}$ & $\begin{array}{c}94.600 \\
101.300\end{array}$ & 95.104 & 0.944 \\
\hline
\end{tabular}




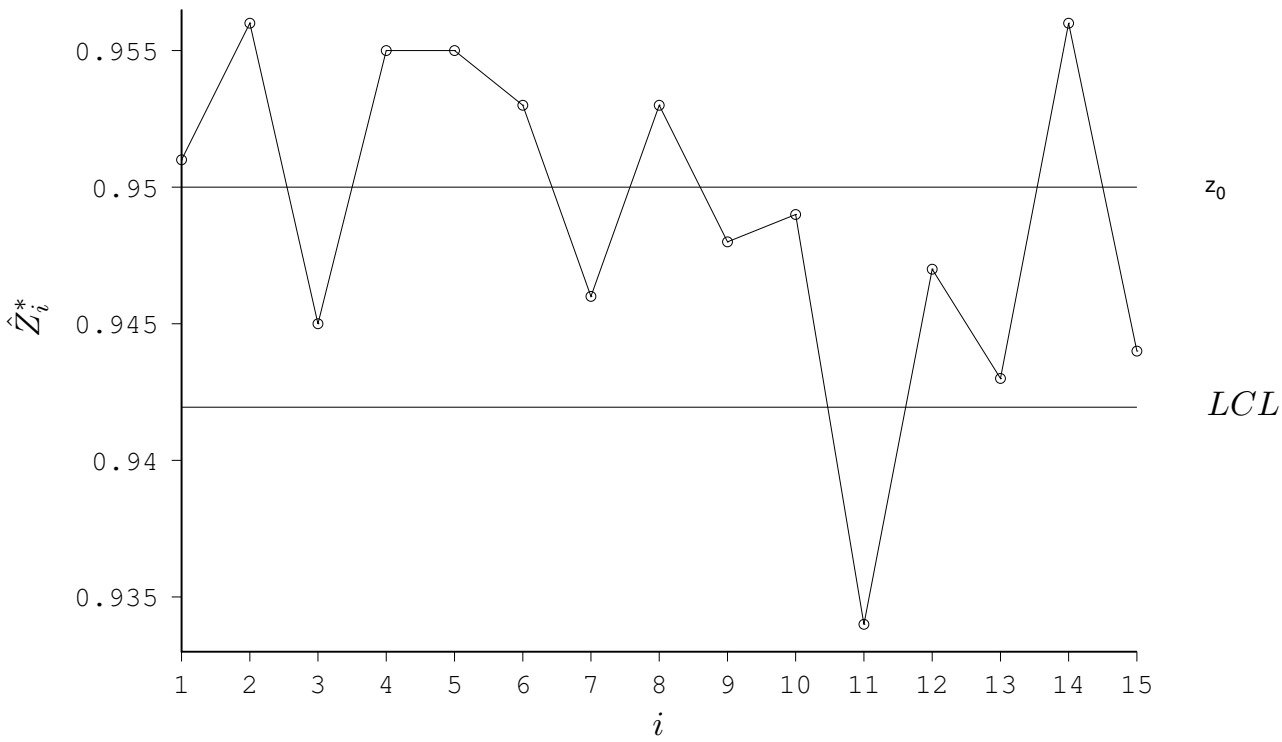

Figure 10: The Shewhart-RZ ${ }^{-}$control chart in the presence of measurement error for the battery recycling industry dataset

[6] D. Brook and D.A. Evans. An approach to the probability distribution of CUSUM run length. Biometrika, 59(3):539-549, 1972.

[7] J.S Hunter. The exponentially weighted moving average. Journal of Quality Technology, 18:203-210, 1986.

[8] K.P. Tran, P. Castagliola, and G. Celano. The performance of the Shewhart-RZ control chart in the presence of measurement error. International Journal of Production Research, 54:7504-7522, 2016.

[9] G. Celano and P. Castagliola. Design of a phase II control chart for monitoring the ratio of two normal variables. Quality and Reliability Engineering International, 32(1):291-308, 2016.

[10] K.P. Tran, P. Castagliola, and G. Celano. Monitoring the ratio of two normal variables using Run Rules type control charts. International Journal of Production Research, 54(6):1670-1688, 2016.

[11] K.P. Tran, P. Castagliola, and G. Celano. Monitoring the ratio of population means of a bivariate normal distribution using CUSUM type control charts. Statistical Papers, 59:387-413, 2016

[12] K.W. Linna, W.H. Woodall, and K.L. Busby. The performance of multivariate control charts in the presence of measurement error. Journal of Quality Technology, 33(3):349-355, 2001. 
[13] A. Tang, P. Castagliola, X. Hu and J. Sun. The performance of the adaptive EWMA median chart in the presence of measurement error. Quality and Reliability Engineering International, 35(1):423-438, 2018.

[14] P.H. Tran, K.P. Tran and A. Rakitzis. A Synthetic median control chart for monitoring the process mean with measurement errors. Quality and Reliability Engineering International, 35(4):1100-1116, 2019.

[15] K. P. Tran, H. D. Nguyen, Q. T. Nguyen, and W. Chattinnawat. Onesided synthetic control charts for monitoring the coefficient of variation with measurement errors. In International conference on industrial engineering and engineering management, pages 1667-1671. IEEE, 2018.

[16] K. P. Tran, H. D. Nguyen, P. H. Tran and C. Heuchenne. On the performance of CUSUM control charts for monitoring the coefficient of variation with measurement errors. The International Journal of Advanced Manufacturing Technology, 104(5-8): 1903-1917, 2019.

[17] K. P. Tran, C. Heuchenne and N. Balakrishnan. On the performance of coefficient of variation charts in the presence of measurement errors. Quality and Reliability Engineering International , 35 (1), 329-350, 2018.

[18] X. Cheng and F. Wang. VSSI median control chart with estimated parameters and measurement errors. Quality and Reliability Engineering International, 34(5):867-881, 2018.

[19] H. Sabahno, A. Amiri and P. Castagliola. Evaluating the effect of measurement errors on the performance of the variable sampling intervals Hotelling's $T^{2}$ control charts. Quality and Reliability Engineering International, 34(8):1785-1799, 2018.

[20] H.D. Nguyen, Q.T. Nguyen, K.P. Tran, and D.P. Ho. On the performance of VSI Shewhart control chart for monitoring the coefficient of variation in the presence of measurement errors. The International Journal of Advanced Manufacturing Technology, 104:211-243, 2019.

[21] F. S. Zaidi, P. Castagliola, K. P. Tran and M. B. C. Khoo. Performance of the hotelling $T^{2}$ control chart for compositional data in the presence of measurement errors. Journal of Applied Statistics, 46(14): 2583-2602, 2019 .

[22] Q. T. Nguyen, K. P. Tran, H. L. Heuchenne, T. H. Nguyen, and H. D. Nguyen. Variable sampling interval Shewhart control charts for monitoring the multivariate coefficient of variation. Applied Stochastic Models in Business and Industry, 2019. 
[23] H. D. Nguyen, K. P. Tran, and T. N. Goh. Variable Sampling Interval Control Charts for Monitoring the Ratio of Two Normal Variables. Journal of Testing and Evaluation, 48, 2019, Doi: 10.1520/JTE20190327.

[24] H. D. Nguyen, K. P. Tran, and H. L. Heuchenne. CUSUM control charts with variable sampling interval for monitoring the ratio of two normal variables. Quality and Reliability Engineering International, 36(2):474-497, 2020.

[25] T. H. Nguyen, H. D. Nguyen, K. D. Tran, T. H. Truong, K. H. Phung, L. H. Nguyen, T. T. N. Le, and K. P. Tran. One-Sided Synthetic-RZ control charts: a new method for anomaly detection. In 6th NAFOSTED conference on Information and Computer Science, pages 262-267. IEEE, 2019.

[26] R.C. Geary. The Frequency Distribution of the Quotient of Two Normal Variates. Journal of the Royal Statistical Society, 93(3):442-446, 1930.

[27] J. Hayya, D. Armstrong, and N. Gressis. A note on the ratio of two normally distributed variables. Management Science, 21(11):1338-1341, 1975.

[28] T. Pham-Gia, N. Turkkan, and E. Marchand. Density of the ratio of two normal random variables and applications. Communications in Statistics - Theory and Methods, 35(9):1569-1591, 2006.

[29] A. Cedilnik, K. Kosmelj, and A. Blejec. The distribution of the ratio of jointly normal variables. Metodoloski Zvezki, 1(1):99-108, 2004.

[30] G. Celano, P. Castagliola, A. Faraz, and S. Fichera. Statistical performance of a control chart for individual observations monitoring the ratio of two normal variables. Quality and Reliability Engineering International, 30(8):1361-1377, 2014.

[31] K.W. Linna and W.H. Woodall. Effect of measurement error on shewart control chart. Jornal of Quality Technology, 33(2):213-222, 2001. 